ESAIM: M2AN 47 (2013) 583-608

DOI: $10.1051 / \mathrm{m} 2 \mathrm{an} / 2012040$
ESAIM: Mathematical Modelling and Numerical Analysis

www.esaim-m2an.org

\title{
OPTIMIZED SCHWARZ METHODS FOR THE BIDOMAIN SYSTEM IN ELECTROCARDIOLOGY
}

\author{
Luca Gerardo-Giorda ${ }^{1}$ and Mauro Perego ${ }^{2}$
}

\begin{abstract}
The propagation of the action potential in the heart chambers is accurately described by the Bidomain model, which is commonly accepted and used in the specialistic literature. However, its mathematical structure of a degenerate parabolic system entails high computational costs in the numerical solution of the associated linear system. Domain decomposition methods are a natural way to reduce computational costs, and Optimized Schwarz Methods have proven in the recent years their effectiveness in accelerating the convergence of such algorithms. The latter are based on interface matching conditions more efficient than the classical Dirichlet or Neumann ones. In this paper we analyze an Optimized Schwarz approach for the numerical solution of the Bidomain problem. We assess the convergence of the iterative method by means of Fourier analysis, and we investigate the parameter optimization in the interface conditions. Numerical results in $2 \mathrm{D}$ and $3 \mathrm{D}$ are given to show the effectiveness of the method.
\end{abstract}

Mathematics Subject Classification. 65M55, 65N30, 92-08.

Received November 29, 2011. Revised July 16, 2012.

Published online January 18, 2013.

\section{INTRODUCTION}

Schwarz algorithms have become increasingly popular over the last decades, due to a widespread availability of more and more powerful distributed computers. The classical Schwarz method was applied to a wide range of partial differential equations, from linear elasticity $[18,19]$ to time harmonic Maxwell's equations [53] and convergence results can be found in several books, see [47, 51, 54], and authoritative reviews, see [5, 59, 60]. The convergence rate of the classical Schwarz method, however, is rather slow and very much dependent on the size of the overlap. Moreover, in general the classical Schwarz method is not convergent in the absence of overlap. To overcome these drawbacks, a new class of Schwarz methods was developed in recent years, namely the optimized Schwarz methods. These methods are based on more effective transmission conditions than the classical Dirichlet conditions at the interfaces between subdomains. Originally, P.L. Lions proposed Robin conditions to obtain convergence without overlap, see [34], while in a short note on non-linear problems [29] Hagstrom et al. suggested nonlocal operators for best performance. In [6], these optimal, non-local transmission conditions were developed for advection-diffusion problems, with local approximations for small viscosity, and

Keywords and phrases. Domain decomposition, optimized schwarz methods, computational electrocardiology.

1 BCAM, Basque Center for Applied Mathematics, Bilbao, Spain. Igerardo@bcamath.org

2 Dept. of Scientific Computing, The Florida State University, Thallahassee, FL, USA. mperego@fsu.edu 
low order frequency approximations were proposed in [13,38]. Optimized transmission conditions for the best performance in a given class of local transmission conditions were introduced for advection diffusion problems in [31], for the Helmholtz equation in [7,24], for Laplace's equation in [17] and for Maxwell's equation [3]. For complete results and attainable performance for symmetric, positive definite problems, see [20], and for time dependent problems, see [21,23]. The Optimized Schwarz methods were also extended to systems of partial differential equations, such as the compressible Euler equations [14] and the full Maxwell system [15]. Recently, Optimized Schwarz strategies have been proposed for the coupling of heterogeneous models, such as in Fluid-Structure Interaction problem [27] and in the coupling of Bidomain and Monodomain models in electrocardiology [28].

The Bidomain model is commonly considered as one of the most complete and accurate models to describe the propagation of the electrical potential in the myocardium tissue, see e.g. $[30,32,46,48,49]$. It consists of a system of nonlinear unsteady partial differential equations including the dynamics of intra and extracellular potentials. The discretization of the Bidomain model is often based on a finite element approximation in space and on implicit-explicit time advancing schemes, that allow to skip the expensive solution of nonlinear systems. The degenerate parabolic nature of this system, however, entails a very ill conditioning for the linear system associated to the Bidomain discretization. In the recent years many efforts have been devoted to the set up of efficient solvers and preconditioners to reduce the high computational costs associated to its numerical solution $[9,10,33,37,41,52,56,57]$, possibly based on multigrid approaches $[39,42,43,50,58]$ or suitable approximations of the equations [26]. Among these works, most are based on a proper decomposition of the computational domain in order to set up parallel preconditioners, or on suitable multigrid schemes still coupled with parallel architectures. In particular, a Classical Schwarz Method coupled with a multigrid approach has been proposed in [39].

In this paper we introduce and analyze an Optimized Schwarz approach to the numerical solution of the Bidomain system in non-symmetric formulation. We rely on Fourier analysis to assess the convergence of the algorithm and to identify the optimal parameters that can be used in the Robin-type interface coupling between the subdomains. This paper can be considered a second step in the presentation of a model adaptive strategy where the costly Bidomain is solved only where actually needed. The computational domain is split into several subdomains, where the Bidomain or the Monodomain models are solved according to a suitable estimator following the wavefront propagation, and where an Optimized Schwarz method is used to couple the subdomain problems [25]. In [28] the convergence of the Optimized Schwarz coupling between Bidomain and Monodomain was proven, while this paper is devoted to the Bidomain-Bidomain coupling.

The paper is organized as follow. In Section 2 the Bidomain system and its numerical approximation are introduced. In Section 3 the Classical Schwarz Method applied to the Bidomain system is described and its convergence properties are enlightened by means of Fourier analysis. In Section 4 the Optimized Schwarz Method for the Bidomain system is introduced and its convergence properties are analyzed, introducing different possible choices for the Robin interface conditions. In Section 5 the use of the Optimized Schwarz Method as a preconditioner for a Krylov solution to the interface problem is introduced. Finally, Section 6 contains numerical tests in two and three dimensions illustrating the convergence properties of the method, and enlightening the parameters combination that provide interface conditions which are robust at once with respect to mesh size and fibers directions.

\section{The Bidomain System AND its NUMERICAL APPROXIMATION}

The cardiac tissue can be represented as a superposition of intra and extracellular media connected by a cell membrane dislocated in the domain. The Bidomain model should take into account the direction of the cardiac fibers. Anatomical studies show that the fibers direction rotates counterclockwise from epicardium to endocardium and that they are arranged in sheets, running across the myocardial wall (see e.g. [49]). We set the problem in a bounded region $\Omega \subset \mathbb{R}^{3}$, and we assume that the cardiac tissue is characterized at each point by three directions: $\boldsymbol{a}_{l}$ along the fiber, $\boldsymbol{a}_{t}$ orthogonal to the fiber direction and in the fiber sheet and $\boldsymbol{a}_{n}$ orthogonal 
to the sheet. The intra and extracellular media present different conductivity values in each direction. We denote by $\sigma_{i}^{l}(\boldsymbol{x})\left(\right.$ resp. $\left.\sigma_{e}^{l}(\boldsymbol{x})\right)$ the intracellular (resp. extracellular) conductivity in $\boldsymbol{a}_{l}(\boldsymbol{x})$ direction at point $\boldsymbol{x} \in \Omega$, and similarly by $\sigma_{i}^{t}(\boldsymbol{x})\left(\sigma_{e}^{t}(\boldsymbol{x})\right)$ and $\sigma_{i}^{n}(\boldsymbol{x})\left(\sigma_{e}^{n}(\boldsymbol{x})\right)$ the conductivities along $\boldsymbol{a}_{t}(\boldsymbol{x})$ and $\boldsymbol{a}_{n}(\boldsymbol{x})$. We will use throughout the paper the notation $\sigma_{\tau}^{l}(\boldsymbol{x}), \sigma_{\tau}^{n}(\boldsymbol{x}), \sigma_{\tau}^{t}(\boldsymbol{x})$ with $\tau=i$,e for indicating intra and extracellular conductivity in a compact form.

The intra and extracellular local anisotropic conductivity tensors read therefore, for $\tau=i, e$,

$$
\mathbf{D}_{\tau}(\boldsymbol{x})=\sigma_{\tau}^{l}(\boldsymbol{x}) \boldsymbol{a}_{l}(\boldsymbol{x}) \boldsymbol{a}_{l}^{T}(\boldsymbol{x})+\sigma_{\tau}^{t}(\boldsymbol{x}) \boldsymbol{a}_{t}(\boldsymbol{x}) \boldsymbol{a}_{t}^{T}(\boldsymbol{x})+\sigma_{\tau}^{n}(\boldsymbol{x}) \boldsymbol{a}_{n}(\boldsymbol{x}) \boldsymbol{a}_{n}^{T}(\boldsymbol{x}) .
$$

Let $u_{i}$ and $u_{e}$ be the intra and extracellular potentials respectively and $u=u_{i}-u_{e}$ be the transmembrane potential. The density current in each domain can be computed as $\mathbf{J}_{\tau}=-\mathbf{D}_{\tau} \nabla u_{\tau}, \tau=i, e$. The net current flux between the intra and the extracellular domain is assumed to be zero as a consequence of the charge conservation in an arbitrary portion of tissue. Denoting by $I_{m}$ the ingoing membrane current flow and by $\chi$ the ratio of membrane area per tissue volume, we get $\nabla \cdot\left(\mathbf{D}_{i} \nabla u_{i}\right)=\chi I_{m}=-\nabla \cdot\left(\mathbf{D}_{e} \nabla u_{e}\right)$, where $I_{m}=C_{m} \mathrm{~d} u / \mathrm{d} t+I_{\text {ion }}(u, \boldsymbol{w})$ being $C_{m}$ a capacitance and $I_{\text {ion }}$ the ionic current, depending on the potential $u$ and on suitable ionic variables that we denote with $\boldsymbol{w}$. The complete Bidomain model reads

$$
\begin{aligned}
\chi C_{m} \partial_{t} u-\nabla \cdot \mathbf{D}_{i} \nabla u_{i}+\chi I_{\text {ion }}(u, \boldsymbol{w}) & =I_{i}^{\text {app }} \\
-\chi C_{m} \partial_{t} u-\nabla \cdot \mathbf{D}_{e} \nabla u_{e}-\chi I_{\text {ion }}(u, \boldsymbol{w}) & =-I_{e}^{\text {app }},
\end{aligned}
$$

where $I_{\text {ion }}(u, \boldsymbol{w})$ is a nonlinear function of the transmembrane potential $u$, specified by a ionic model, and where $I_{i, e}^{\text {app }}$ are applied external stimuli. The problem is completed by initial conditions, $u_{i}(\boldsymbol{x}, 0)=u_{i, 0}, u_{e}(\boldsymbol{x}, 0)=$ $u_{e, 0}, u(\boldsymbol{x}, 0)=u_{i, 0}-u_{e, 0}$ and by homogeneous Neumann boundary conditions on $\partial \Omega$, modeling an insulated myocardium,

$$
\mathbf{n}^{T} \mathbf{D}_{i} \nabla u_{i}(\boldsymbol{x}, t)=0 \quad \text { and } \quad \mathbf{n}^{T} \mathbf{D}_{e} \nabla u_{e}(\boldsymbol{x}, t)=0, \quad \text { on } \partial \Omega \times(0, T),
$$

where $\mathbf{n}$ is the unit normal outward-pointing vector on the surface. As a consequence of the Gauss theorem, the applied external stimuli must fulfill the compatibility condition

$$
\int_{\Omega} I_{i}^{\mathrm{app}} \mathrm{d} \boldsymbol{x}=\int_{\Omega} I_{\mathrm{e}}^{\mathrm{app}} \mathrm{d} \boldsymbol{x} .
$$

System (2.2) consists of two parabolic reaction diffusion equations for $u_{i}$ and $u_{e}$ where the vector of time derivatives is multiplied by a singular matrix. The system is thus said to be degenerate. The transmembrane potential $u$ is uniquely determined, while the intra and extracellular potentials $u_{i}$ and $u_{e}$ are determined up to the same function of time, whose value is usually obtained by imposing that $u_{e}$ has zero mean on $\Omega$. For well-posedeness analysis of the Bidomain problem see [12] (Fitzhugh-Nagumo model) and [55] (Luo-Rudy I model).

In what follows we will rely on a non-symmetric formulation of (2.2) (see e.g. [26]). By linear combinations of the two equations in $(2.2)$, with coefficients $\left(\frac{\lambda}{1+\lambda},-\frac{1}{1+\lambda}\right)$, and $(1,1)$, Bidomain system can be reformulated in terms of the transmembrane and the extracellular potentials $u$ and $u_{e}$. We let $\boldsymbol{u}=\left(u, u_{e}\right)^{T}$, and defining

$$
\mathbf{D}=\left[\begin{array}{cc}
\frac{\lambda}{1+\lambda} \mathbf{D}_{i} & \frac{\lambda}{1+\lambda} \mathbf{D}_{i}-\frac{1}{1+\lambda} \mathbf{D}_{e} \\
\mathbf{D}_{i} & \mathbf{D}_{i}+\mathbf{D}_{e}
\end{array}\right], \quad \mathbf{D}_{B}=\left[\begin{array}{cc}
\mathbf{D}_{i} & \mathbf{D}_{i} \\
\mathbf{0} & \mathbf{D}_{e}
\end{array}\right], \quad \mathbf{E}_{1}=\left[\begin{array}{ll}
1 & 0 \\
0 & 0
\end{array}\right], \quad \mathbf{e}_{1}=\left[\begin{array}{l}
1 \\
0
\end{array}\right]
$$

the non-symmetric Bidomain reads

$$
\left\{\begin{array}{l}
\chi C_{m} \mathbf{E}_{1} \partial_{t} \boldsymbol{u}-\nabla \cdot \mathbf{D} \nabla \boldsymbol{u}+\chi I_{\mathrm{ion}}(\boldsymbol{u}, \boldsymbol{w}) \boldsymbol{e}_{1}=\boldsymbol{I}^{\mathrm{app}} \\
\boldsymbol{u}(\boldsymbol{x}, 0)=\left.\boldsymbol{u}_{0}(\boldsymbol{x}) \quad \boldsymbol{n}^{T} \mathbf{D}_{B} \nabla \boldsymbol{u}\right|_{\partial \Omega}=0,
\end{array}\right.
$$


where we have $\boldsymbol{I}^{\mathrm{app}}=\left(\frac{\lambda I_{i}^{\mathrm{app}}+I_{e}^{\mathrm{app}}}{1+\lambda}, I_{i}^{\mathrm{app}}-I_{e}^{\mathrm{app}}\right)^{T}$. The motivation for this choice is twofold. On the one hand, in view of a model adaptive strategy, the presence of the membrane potential $u$ in the non symmetric formulation naturally fits in the coupling with the Monodomain model. On the other hand, the parabolic-elliptic formulation (2.5) is widely used in the Bioengineering community, and features better scalability properties in a parallel settings when block-preconditioners are employed [40].

In the rest of the paper, following [28], we choose $\lambda=\sigma_{\mathrm{e}}^{l} / \sigma_{i}^{l}$, and the conductivity matrix reads

$$
\mathbf{D}=\left[\begin{array}{cc}
\frac{\sigma_{\mathrm{e}}^{l}}{\sigma_{i}^{l}+\sigma_{\mathrm{e}}^{l}} \mathbf{D}_{i} & \frac{\sigma_{\mathrm{e}}^{l}}{\sigma_{i}^{l}+\sigma_{\mathrm{e}}^{l}} \mathbf{D}_{i}-\frac{\sigma_{i}^{l}}{\sigma_{i}^{l}+\sigma_{\mathrm{e}}^{l}} \mathbf{D}_{e} \\
\mathbf{D}_{i} & \mathbf{D}_{i}+\mathbf{D}_{e}
\end{array}\right]
$$

\subsection{Numerical approximation}

We give a quick glance to the numerical approximation of the Bidomain model. Since this is a well established subject, we do not dwell much on this topic, but for a more detailed description we refer the interested reader to e.g. $[10,11,57]$.

\subsubsection{Time integration}

Let us assume to use a fixed time step $\Delta t$, even if time adaptive schemes have been considered as well (see e.g. $[10,44]$ ). We denote with superscript $n$ the variables computed at time $t^{n}=n \Delta t$. The Bidomain equations (2.5) can be advanced in time by a semi-implicit scheme. The ionic variables $\boldsymbol{w}$ are integrated exactly in time upon an appropriate linearization around the membrane potential at the previous time step (see e.g. [44]). The time step is selected to guarantee stability to the time advancing scheme. In what follows we do not rely on a specific choice for the ionic model describing the cell membrane currents. More precisely, for $0<n \leq N=T / \Delta t$, moving from $t^{n}$ to $t^{n+1}$, we solve in $\Omega$

$$
\left\{\begin{array}{l}
\chi C_{m} \mathbf{E}_{1} \frac{\boldsymbol{u}^{n+1}-\boldsymbol{u}^{n}}{\Delta t}-\nabla \cdot \mathbf{D} \nabla \boldsymbol{u}^{n+1}=\boldsymbol{I}^{\mathrm{app}}-\chi I_{\mathrm{ion}}\left(\boldsymbol{u}^{n}, \boldsymbol{w}^{n+1}\right) \boldsymbol{e}_{1} \\
\boldsymbol{u}^{0}(\boldsymbol{x})=\left.\boldsymbol{u}_{0}(\boldsymbol{x}) \quad \boldsymbol{n}^{T} \mathbf{D}_{B} \nabla \boldsymbol{u}^{n+1}\right|_{\partial \Omega}=0 .
\end{array}\right.
$$

In the sequel we let

$$
\boldsymbol{f}^{n+1}=\frac{\chi C_{m}}{\Delta t} \mathbf{E}_{1} \boldsymbol{u}^{n}+\boldsymbol{I}^{\mathrm{app}}-\chi I_{\mathrm{ion}}\left(\boldsymbol{u}^{n}, \boldsymbol{w}^{n+1}\right) \boldsymbol{e}_{1},
$$

and we drop any index referring to time discretization as long as the context is clear.

\subsubsection{Space discretization}

We discretize in space the domain with a regular triangulation $\mathcal{T}_{h}$ and we consider a finite element space $V_{h}$, in which we will look for the approximate solutions, that we denote by $u^{h}$ and $u_{e}^{h}$. For the numerical tests in Section 6, we chose $V_{h}$ as the space of piecewise linear continuous functions on $\mathcal{T}_{h}$, but we claim that other finite element spaces can be used $[10,57]$.

We denote by $\Phi=\left\{\varphi_{j}\right\}_{j=1}^{N_{h}}$ a basis for $V_{h}$, by $\mathcal{K}_{\tau}(\tau=i, e)$ the stiffness matrix with $\mathcal{K}_{\tau}^{i j}=$ $\left.\sum_{K \in \mathcal{T}_{h}}\left(\mathbf{D}_{\tau} \nabla \varphi_{j}, \nabla \varphi_{i}\right)\right|_{K}$, and by $\mathcal{M}$ the mass matrix with entries $\mathcal{M}^{i j}=\left.\sum_{K \in \mathcal{T}_{h}}\left(\varphi_{j}, \varphi_{i}\right)\right|_{K}, \varphi_{i}, \varphi_{j} \in \Phi$. The unknowns of the fully discrete problem are represented by vectors $\mathbf{u}$ and $\mathbf{u}_{e}$, storing the nodal values of $u^{h}$ and $u_{e}^{h}$, respectively, and we let $\mathbf{f}$ denote the discretization of the forcing term $\boldsymbol{f}$.

At step $t^{n+1}$ the discrete Bidomain model solves

$$
\mathbf{B} \mathcal{U}^{n+1}=\mathbf{f}^{n+1}, \quad \mathbf{B}=\left[\begin{array}{ll}
\frac{\chi C_{m}}{\Delta t} \mathcal{M}+\frac{\sigma_{\mathrm{e}}^{l} \mathcal{K}_{i}}{\sigma_{i}^{l}+\sigma_{\mathrm{e}}^{l}} & \frac{\sigma_{\mathrm{e}}^{l} \mathcal{K}_{i}}{\sigma_{i}^{l}+\sigma_{\mathrm{e}}^{l}}-\frac{\sigma_{i}^{l} \mathcal{K}_{e}}{\sigma_{i}^{l}+\sigma_{\mathrm{e}}^{l}} \\
\mathcal{K}_{i} & \mathcal{K}_{i}+\mathcal{K}_{e}
\end{array}\right], \quad \mathcal{U}=\left[\begin{array}{l}
\mathbf{u} \\
\mathbf{u}_{e}
\end{array}\right] .
$$




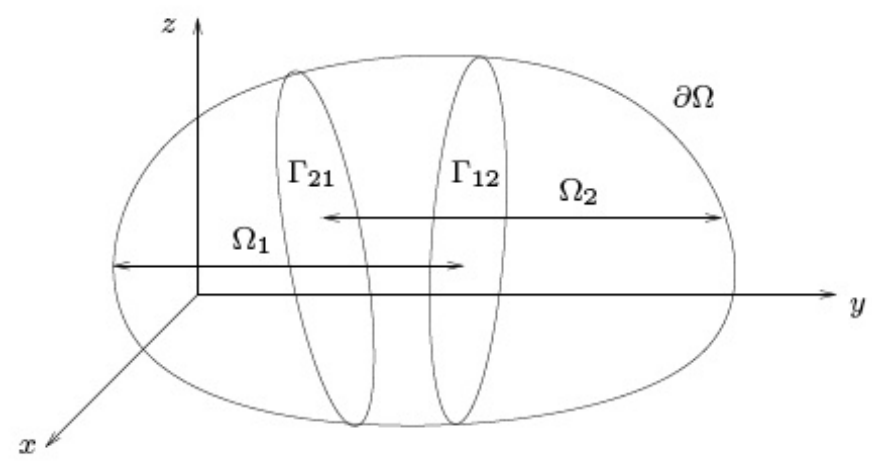

FiguRE 1. Overlapping domain decomposition.

Since the Bidomain system (2.2) is degenerate, the matrix B in its discrete formulation is singular, with a kernel spanned by the constants. We thus solve (2.7) with an iterative method (GMRES, due to its lack of symmetry) and we force a zero mean value on the extracellular potential by imposing $\mathbf{1}^{T} \mathcal{M} \mathbf{u}_{e}=0$.

\section{Classical Schwarz Method}

In order to present the classical Schwarz Algorithm, we decompose the computational domain into two overlapping subdomains as illustrated in Figure 1. We choose to describe the formulation in a two-domain settings for sake of simplicity in presentation, but the extension of the algorithm formulation to an arbitrary number of subdomains does not present any conceptual difficulties. We denote with $u_{i, l}, u_{e, l}$, and $u_{l}=u_{i, l}-u_{e, l}$ $(l=1,2)$ the intracellular, extracellular and transmembrane potentials in $\Omega_{1}$ and $\Omega_{2}$, respectively. The unknowns are collected in the vectors $\boldsymbol{u}_{l}=\left(u_{l}, u_{e, l}\right)^{T}(l=1,2)$. At each time step, the classical alternating Schwarz algorithm enforces Dirichlet continuities on the interfaces and reads as follows.

Given $\boldsymbol{u}_{2}^{0}$ on $\Gamma_{12}$, solve for $p \geq 0$ until convergence

$$
\begin{array}{ll}
\frac{\chi C_{m}}{\Delta t} \mathbf{E}_{1} \boldsymbol{u}_{1}^{p+1}-\nabla \cdot \mathbf{D} \nabla \boldsymbol{u}_{1}^{p+1}=\boldsymbol{f}_{1} & \text { in } \Omega_{1} \\
\boldsymbol{n}_{1}^{T} \mathbf{D}_{B} \nabla \boldsymbol{u}_{1}^{p+1}=0 & \text { on } \partial \Omega_{1} \cap \partial \Omega \\
\boldsymbol{u}_{1}^{p+1}=\boldsymbol{u}_{2}^{p} & \text { on } \Gamma_{12} \\
\frac{\chi C_{m}}{\Delta t} \mathbf{E}_{1} \boldsymbol{u}_{2}^{p+1}-\nabla \cdot \mathbf{D} \nabla \boldsymbol{u}_{2}^{p+1}=\boldsymbol{f}_{2} & \text { in } \Omega_{2} \\
\boldsymbol{n}_{2}^{T} \mathbf{D}_{B} \nabla \boldsymbol{u}_{1}^{p+1}=0 & \text { on } \partial \Omega_{2} \cap \partial \Omega \\
\boldsymbol{u}_{2}^{p+1}=\boldsymbol{u}_{1}^{p+1} & \text { on } \Gamma_{21} .
\end{array}
$$

The Classical Schwarz Algorithm above can be easily parallelized by choosing $\boldsymbol{u}_{1}^{p}$ instead of $\boldsymbol{u}_{1}^{p+1}$ on $\Gamma_{21}$. In such case also an initial value $\boldsymbol{u}_{1}^{0}$ has to be provided on $\Gamma_{21}$.

\subsection{Convergence analysis}

We analyze here the convergence properties of the classical Schwarz algorithm via Fourier analysis, and to this aim we consider throughout this section the problem on the infinite domain $\Omega=\mathbb{R}^{3}$, decomposed into

$$
\Omega_{1}=(-\infty, L) \times \mathbb{R}^{2}, \quad \Omega_{2}=(0, \infty) \times \mathbb{R}^{2},
$$


where the interfaces are $\Gamma_{12}=\{L\} \times \mathbb{R}^{2}$ and $\Gamma_{21}=\{0\} \times \mathbb{R}^{2}$, and the overlap size is $L \geq 0$. We disregard here the boundary conditions and require the solutions to be bounded at infinity. Notice that the asymptotic requirements for the Fourier transformability of the extracellular potential automatically fix the arbitrary function of time. We assume axial symmetry of the fibers, and the longitudinal axis of the fibers (the principal direction of the action potential propagation) to be orthogonal to the interface $\Gamma$, so that the diffusion tensors are given by

$$
\mathbf{D}_{i}=\left[\begin{array}{ll}
\sigma_{i}^{l} & \\
& \sigma_{i}^{t} \mathbf{I}_{2}
\end{array}\right] \quad \mathbf{D}_{e}=\left[\begin{array}{ll}
\sigma_{\mathrm{e}}^{l} & \\
& \sigma_{\mathrm{e}}^{t} \mathbf{I}_{2}
\end{array}\right]
$$

being $\mathbf{I}_{2}$ the $2 \times 2$ identity matrix.

We apply a Fourier transform in the $y$ and $z$ directions, which is defined, for any $w(x, y, z) \in L^{2}\left(\mathbb{R}^{3}\right)$, as

$$
\mathcal{F}: w(x, y, z) \mapsto \widehat{w}\left(x, k_{y}, k_{z}\right)=\iint_{\mathbb{R}^{2}} \mathrm{e}^{-i\left(k_{y} y+k_{z} z\right)} w(x, y, z) \mathrm{d} y \mathrm{~d} z,
$$

where we denote by $k_{y}$ and $k_{z}$ the dual variables. We note in the sequel $\boldsymbol{k}^{2}=k_{y}^{2}+k_{z}^{2}$, and we can quantify in the frequency space, without loss of generality, the error on the interface $\Gamma_{12}$ at the $p$-th iteration as $\varepsilon^{p}=$ $\widehat{\boldsymbol{u}}_{2}^{p}(L, \boldsymbol{k})-\widehat{\boldsymbol{u}}_{2}(L, \boldsymbol{k})$. This allows us to define the reduction factor of the Schwarz algorithm at the $(p+1)$-th iteration as the spectral radius of the iteration matrix mapping $\varepsilon^{p}$ into $\varepsilon^{p+1}$. Since the time discretized problem is linear, it is enough to analyze the convergence to the zero solution in the absence of forcing terms (i.e. for $\left.\boldsymbol{f}_{1}=\boldsymbol{f}_{2}=0\right)$.

In the Fourier space, the Bidomain equation within $\Omega_{j}(j=1,2)$ is given by (see also [28])

$$
\left[\begin{array}{cc}
\frac{\sigma_{i}^{l} \sigma_{\mathrm{e}}^{l}}{\sigma_{i}^{l}+\sigma_{\mathrm{e}}^{l}} & 0 \\
\sigma_{i}^{l} & \sigma_{i}^{l}+\sigma_{\mathrm{e}}^{l}
\end{array}\right] \partial_{x x}\left[\begin{array}{c}
\widehat{u}_{j} \\
\widehat{u}_{e, j}
\end{array}\right]=\left[\begin{array}{c}
\frac{\chi C_{m}}{\Delta t}+\frac{\sigma_{\mathrm{e}}^{l}}{\sigma_{i}^{l}+\sigma_{\mathrm{e}}^{l}} \sigma_{i}^{t} \boldsymbol{k}^{2} \frac{\sigma_{\mathrm{e}}^{l} \sigma_{i}^{t}-\sigma_{\mathrm{e}}^{t} \sigma_{i}^{l}}{\sigma_{i}^{l}+\sigma_{\mathrm{e}}^{l}} \boldsymbol{k}^{2} \\
\sigma_{i}^{t} \boldsymbol{k}^{2}
\end{array}\right]\left[\begin{array}{c}
\left.\widehat{u}_{j}^{t}+\sigma_{\mathrm{e}}^{t}\right) \boldsymbol{k}^{2} \\
\widehat{u}_{e, j}
\end{array}\right],
$$

and a simple algebra yields

$$
\partial_{x x} \widehat{\boldsymbol{u}}_{j}=A(\boldsymbol{k}) \widehat{\boldsymbol{u}}_{j} \quad A(\boldsymbol{k})=\left[\begin{array}{c}
\frac{\chi C_{m}}{\Delta t}\left(\frac{1}{\sigma_{i}^{t}}+\frac{1}{\sigma_{e}^{\tau}}\right)+\frac{\sigma_{i}^{t}}{\sigma_{i}^{t}} \boldsymbol{k}^{2}\left(\frac{\sigma_{i}^{t}}{\sigma_{i}^{i}}-\frac{\sigma_{e}^{t}}{\sigma_{e}^{t}}\right) \boldsymbol{k}^{2} \\
-\frac{\chi C_{m}}{\Delta t} \frac{1}{\sigma_{e}^{\tau}} \\
\frac{\sigma_{e}^{t}}{\sigma_{e}^{t}} \boldsymbol{k}^{2}
\end{array}\right] .
$$

Proposition 3.1. For $L>0$ the Classical Schwarz Algorithm (3.1) converges for every initial guess and for every Fourier mode $\boldsymbol{k}>0$. Moreover, its reduction factor is independent of the iteration and is given by

$$
\rho_{u}^{\mathrm{Cla}}(\boldsymbol{k}, L)=\mathrm{e}^{-2 \sqrt{\eta^{+}(\boldsymbol{k})} L},
$$

for the error in $u$, and by

$$
\rho_{u_{e}}^{\mathrm{Cla}}(\boldsymbol{k}, L)=\mathrm{e}^{-2 \sqrt{\eta^{-}(\boldsymbol{k})} L},
$$

for the error in $u_{e}$, where $\eta^{ \pm}(\boldsymbol{k})$ are the eigenvalues of the $2 \times 2$ matrix $A(\boldsymbol{k})$ defined in (3.3).

Proof. Given $\widehat{\boldsymbol{u}}_{2}^{p}$ on $\{\boldsymbol{k} \in \mathbb{R}, x \geq 0\}$, the $(p+1)$-th iteration of the classical Schwarz Algorithm solves in the Fourier space

$$
\begin{aligned}
& \partial_{x x} \widehat{\boldsymbol{u}}_{1}^{p+1}=A(\boldsymbol{k}) \widehat{\boldsymbol{u}}_{1}^{p+1} \boldsymbol{k} \in \mathbb{R}, x<L \quad \partial_{x x} \widehat{\boldsymbol{u}}_{2}^{p+1}=A(\boldsymbol{k}) \widehat{\boldsymbol{u}}_{2}^{p+1} \boldsymbol{k} \in \mathbb{R}, x>0 \\
& \widehat{\boldsymbol{u}}_{1}^{p+1}=\widehat{\boldsymbol{u}}_{2}^{p} \quad \boldsymbol{k} \in \mathbb{R}, x=L \quad \widehat{\boldsymbol{u}}_{2}^{p+1}=\widehat{\boldsymbol{u}}_{1}^{p+1} \quad \boldsymbol{k} \in \mathbb{R}, x=0 .
\end{aligned}
$$

In both subdomains, we solve Bidomain system by diagonalizing the matrix $A(\boldsymbol{k})=W(\boldsymbol{k}) \mathrm{H}(\boldsymbol{k}) W^{-1}(\boldsymbol{k})$ and introducing the characteristic variables $\widehat{\boldsymbol{z}}^{p+1}=W^{-1}(\boldsymbol{k}) \widehat{\boldsymbol{u}}^{p+1}$, namely

$$
\left\{\begin{array}{l}
\partial_{x x} \widehat{\boldsymbol{z}}^{p+1}=\mathrm{H}(\boldsymbol{k}) \widehat{\boldsymbol{z}}^{p+1} \\
\widehat{\boldsymbol{u}}^{p+1}=W(\boldsymbol{k}) \widehat{\boldsymbol{z}}^{p+1}
\end{array}, \quad \mathrm{H}(\boldsymbol{k})=\left[\begin{array}{cc}
\eta^{+}(\boldsymbol{k}) & 0 \\
0 & \eta^{-}(\boldsymbol{k})
\end{array}\right], \quad W(\boldsymbol{k})=\left[\begin{array}{ll}
\boldsymbol{w}^{+}(\boldsymbol{k}) & \boldsymbol{w}^{-}(\boldsymbol{k})
\end{array}\right] .\right.
$$


The eigenvalues of $A(\boldsymbol{k})$ are

$$
\begin{aligned}
\eta^{ \pm}(\boldsymbol{k})= & \frac{1}{2}\left[\frac{1}{\sigma_{i}^{l}}\left(\frac{\chi C_{m}}{\Delta t}+\sigma_{i}^{t} \boldsymbol{k}^{2}\right)+\frac{1}{\sigma_{\mathrm{e}}^{l}}\left(\frac{\chi C_{m}}{\Delta t}+\sigma_{\mathrm{e}}^{t} \boldsymbol{k}^{2}\right)\right] \\
& \pm \frac{1}{2} \sqrt{\left[\frac{1}{\sigma_{i}^{l}}\left(\frac{\chi C_{m}}{\Delta t}+\sigma_{i}^{t} \boldsymbol{k}^{2}\right)-\frac{1}{\sigma_{\mathrm{e}}^{l}}\left(\frac{\chi C_{m}}{\Delta t}+\sigma_{\mathrm{e}}^{t} \boldsymbol{k}^{2}\right)\right]^{2}+\frac{4}{\sigma_{i}^{l} \sigma_{\mathrm{e}}^{l}}\left[\frac{\chi C_{m}}{\Delta t}\right]^{2}},
\end{aligned}
$$

while the corresponding eigenvectors are

$$
\boldsymbol{w}^{+}(\boldsymbol{k})=\left[\begin{array}{c}
A_{12}(\boldsymbol{k}) \\
\eta^{+}(\boldsymbol{k})-A_{11}(\boldsymbol{k})
\end{array}\right] \quad \boldsymbol{w}^{-}(\boldsymbol{k})=\left[\begin{array}{c}
A_{12}(\boldsymbol{k}) \\
\eta^{-}(\boldsymbol{k})-A_{11}(\boldsymbol{k})
\end{array}\right] .
$$

Since $\operatorname{det} A(\boldsymbol{k})>0$ and $[\operatorname{tr} A(\boldsymbol{k})]^{2}-4 \operatorname{det} A(\boldsymbol{k})>0$, both eigenvalues $\eta^{ \pm}(\boldsymbol{k})$ are real positive. The condition at infinity excludes growing solutions as $x \rightarrow \pm \infty$, thus the general solution in $\Omega_{1}$ at the $(p+1)$-th iteration is given by

$$
\widehat{\boldsymbol{u}}_{1}^{p+1}(x, \boldsymbol{k})=\beta_{1}^{p+1} \boldsymbol{w}^{+}(\boldsymbol{k}) \mathrm{e}^{\sqrt{\eta^{+}(\boldsymbol{k})}(x-L)}+\beta_{2}^{p+1} \boldsymbol{w}^{-}(\boldsymbol{k}) \mathrm{e}^{\sqrt{\eta^{-}(\boldsymbol{k})}(x-L)},
$$

while the general solution in $\Omega_{2}$ at the $(p+1)$-th iteration is given by

$$
\widehat{\boldsymbol{u}}_{2}^{p+1}(x, \boldsymbol{k})=\gamma_{1}^{p+1} \boldsymbol{w}^{+}(\boldsymbol{k}) \mathrm{e}^{-\sqrt{\eta^{+}(\boldsymbol{k})} x}+\gamma_{2}^{p+1} \boldsymbol{w}^{-}(\boldsymbol{k}) \mathrm{e}^{-\sqrt{\eta^{-}(\boldsymbol{k})} x} .
$$

The vectors $\boldsymbol{\beta}^{p+1}=\left(\beta_{1}^{p+1}, \beta_{2}^{p+1}\right)$ and $\gamma^{p+1}=\left(\gamma_{1}^{p+1}, \gamma_{2}^{p+1}\right)$ are then uniquely determined by the boundary conditions on the interfaces $\{x=0\}$ and $\{x=L\}$.

Owing to (3.6), we get

$$
\beta_{1}^{p+1} \boldsymbol{w}^{+}(\boldsymbol{k})+\beta_{2}^{p+1} \boldsymbol{w}^{-}(\boldsymbol{k})=\gamma_{1}^{p} \boldsymbol{w}^{+}(\boldsymbol{k}) \mathrm{e}^{-\sqrt{\eta^{+}(\boldsymbol{k})} L}+\gamma_{2}^{p} \boldsymbol{w}^{-}(\boldsymbol{k}) \mathrm{e}^{-\sqrt{\eta^{-}(\boldsymbol{k})} L}
$$

and

$$
\gamma_{1}^{p+1} \boldsymbol{w}^{+}(\boldsymbol{k})+\gamma_{2}^{p+1} \boldsymbol{w}^{-}(\boldsymbol{k})=\beta_{1}^{p+1} \boldsymbol{w}^{+}(\boldsymbol{k}) \mathrm{e}^{-\sqrt{\eta^{+}(\boldsymbol{k})} L}+\beta_{2}^{p+1} \boldsymbol{w}^{-}(\boldsymbol{k}) \mathrm{e}^{-\sqrt{\eta^{-}(\boldsymbol{k})} L},
$$

that, by defining,

$$
\Lambda(\boldsymbol{k}, L)=\left[\begin{array}{cc}
\mathrm{e}^{-\sqrt{\eta^{+}(\boldsymbol{k})} L} & 0 \\
0 & \mathrm{e}^{-\sqrt{\eta^{-}(\boldsymbol{k})} L}
\end{array}\right]
$$

can be rewritten in matrix form as

$$
W(\boldsymbol{k}) \boldsymbol{\beta}^{p+1}=W(\boldsymbol{k}) \Lambda(\boldsymbol{k}, L) \boldsymbol{\gamma}^{p} \quad W(\boldsymbol{k}) \boldsymbol{\gamma}^{p+1}=W(\boldsymbol{k}) \Lambda(\boldsymbol{k}, L) \boldsymbol{\beta}^{p+1} .
$$

Since $W(\boldsymbol{k})$ is invertible for $\boldsymbol{k}>0$, we get $\boldsymbol{\gamma}^{p+1}=\Lambda^{2}(\boldsymbol{k}, L) \boldsymbol{\gamma}^{p}$, and, given $\boldsymbol{\gamma}^{0}$,

$$
\boldsymbol{\gamma}^{p}=\Lambda^{2 p}(\boldsymbol{k}, L) \boldsymbol{\gamma}^{0}
$$

The global reduction factor of the Classical Schwarz Algorithm is thus given by the spectral radius of the matrix $\Lambda^{2}(\boldsymbol{k}, L)$

$$
\rho^{\mathrm{Cla}}(\boldsymbol{k}, L)=\max \left(\mathrm{e}^{-2 \sqrt{\eta^{+}(\boldsymbol{k})} L}, \mathrm{e}^{-2 \sqrt{\eta^{-}(\boldsymbol{k})} L}\right)=\mathrm{e}^{-2 \sqrt{\eta^{-}(\boldsymbol{k})} L}
$$

Owing to (3.8), the last equality follows. The diagonal form of the matrix $\Lambda(\boldsymbol{k}, L)$, however, allows us to identify a reduction factor for the error in the $u$ variable and one for the $u_{e}$ variable. In particular we have

$$
\rho_{u}^{\mathrm{Cla}}(\boldsymbol{k}, L)=\mathrm{e}^{-2 \sqrt{\eta^{+}(\boldsymbol{k})} L} \quad \rho_{u_{e}}^{\mathrm{Cla}}(\boldsymbol{k}, L)=\mathrm{e}^{-2 \sqrt{\eta^{-}(\boldsymbol{k})} L},
$$

which concludes the proof. 

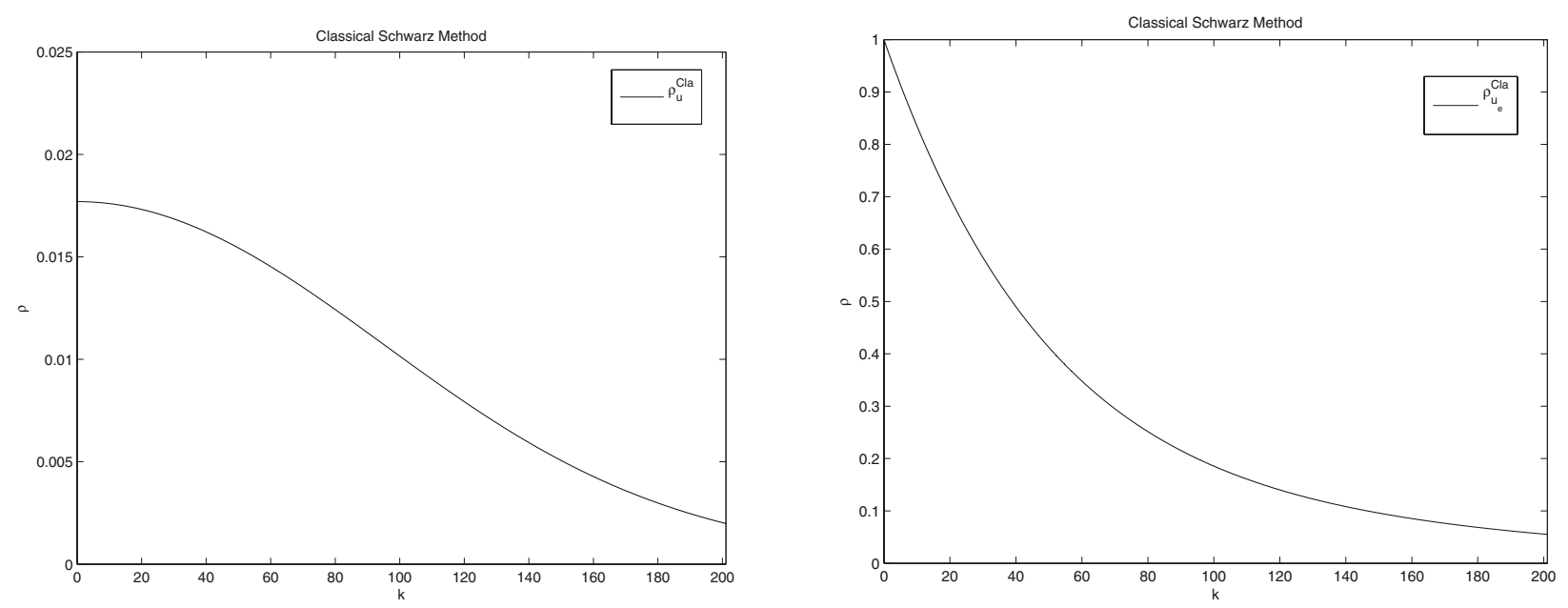

Figure 2. Reduction factors $\rho_{u}^{\text {Cla }}(\boldsymbol{k}, L)$ (left) and $\rho_{u_{e}}^{\text {Cla }}(\boldsymbol{k}, L)$ (right) as a function of the frequency $\boldsymbol{k}$ for the Classical Schwarz Algorithm with minimal overlapping decompositions $(L=h)$. Notice the different scales used.

Remark 3.2. The definition of the reduction factors for the classical Schwarz algorithm (3.4) and (3.5) entails that overlap is mandatory for convergence for all Fourier mode $\boldsymbol{k}>0$. Moreover, since $\boldsymbol{\eta}^{-}(0)=0, \rho_{u_{e}}^{\mathrm{Cla}}(0, L)=1$ regardless of the overlap size, and the iterative form of the classical Schwarz algorithm does not converge in the extracellular potential for the frequency $\boldsymbol{k}=0$, corresponding to constant solutions along the interface. This is a consequence of the degenerate nature of the Bidomain system: its kernel in the non-symmetric formulation is spanned by the vector $[\mathbf{0}, \mathbf{1}]$ and the vaule of $u_{e}$ is known only up to a constant. This problem can be dealt with by forcing at each iteration a zero mean on the extracellular potentials $u_{e, l}(l=1,2)$, which fixes the arbitrary constant in the solution. Also, another option is to use the classical Schwarz algorithm as a preconditioner for a Krylov method, which can handle isolated problems in the spectrum. This last approach is actually the most popular in the literature $[3,15,20,24]$.

We plot in Figure 2 the reduction factors $\rho_{u}^{\text {Cla }}(\boldsymbol{k}, L)$ and $\rho_{u_{e}}^{\text {Cla }}(\boldsymbol{k}, L)$ as a function of $\boldsymbol{k}$, for a mesh size of order $h=1 / 64$ (reasonable in applications [8]), and a minimal overlap of size $L=h$ : the values of $\sigma_{i}^{\tau}$ and $\sigma_{\mathrm{e}}^{\tau}$ $(\tau=l, t)$ are the ones proposed in [10]. Notice the different scale in the figures: we expect the algorithm to converge much faster on $u$ than on $u_{e}$, as the latter is associated with the singular part of the operator.. Such theoretical conclusion will be supported by numerical evidence in Section 6. We point out that enforcing a zero mean on the extracellular potential to fix the arbitrary constant does not help in terms of convergence. Such procedure is an instance of the null-space method. It is known that, for pure elliptic problems with homogeneous Neumann boundary conditions (which is in practice the case of the equation for the extracellular potential $u_{e}$ ), the condition number of the resulting matrices are larger than the effective condition number of the singular matrix [4], and this would eventually increase the iterations counts in the solution of the associated linear system.

\section{Optimized Schwarz Method}

We introduce here a modification of the classical Dirichlet interface conditions, which lead to Optimized Schwarz Methods. Such methods have become very popular in the last decade [15,20,24], and are a generalization of the non-overlapping Robin-Robin algorithm proposed by P.L. Lions in [34], that ensures convergence also without relaxation. 
We first derive suitable coupling conditions at the interfaces. We focus on $\Gamma_{12}$, but the adaptation to $\Gamma_{21}$ is straightforward. Let again $u_{i, l}, u_{e, l}$, and $u_{l}=u_{i, l}-u_{e, l}(l=1,2)$ denote the intracellular, extracellular and transmembrane potential in $\Omega_{1}$ and $\Omega_{2}$, respectively.

Optimized Schwarz Methods are based upon interface continuity requirements on traces and fluxes of Robin type. In this case, the coupling of intracellular and extracellular potentials and fluxes along $\Gamma_{12}$ is given by

$$
\boldsymbol{n}_{1}^{T} \mathbf{D}_{i} \nabla u_{i, 1}+\alpha u_{i, 1}=\boldsymbol{n}_{1}^{T} \mathbf{D}_{i} \nabla u_{i, 2}+\alpha u_{i, 2}, \quad \boldsymbol{n}_{1}^{T} \mathbf{D}_{e} \nabla u_{e, 1}+\beta u_{e, 1}=\boldsymbol{n}_{1}^{T} \mathbf{D}_{e} \nabla u_{e, 2}+\beta u_{e, 2},
$$

for $\alpha, \beta>0$. The same linear combinations yielding to the non-symmetric formulation (2.5), applied to (4.1) provide the following equivalent coupling conditions on $\Gamma_{12}$,

$$
\begin{gathered}
\boldsymbol{n}_{1}^{T} \frac{\sigma_{\mathrm{e}}^{l} \mathbf{D}_{i}}{\sigma_{i}^{l}+\sigma_{\mathrm{e}}^{l}}\left(\nabla u_{1}+\nabla u_{e, 1}\right)-\boldsymbol{n}_{1}^{T} \frac{\sigma_{i}^{l} \mathbf{D}_{e}}{\sigma_{i}^{l}+\sigma_{\mathrm{e}}^{l}} \nabla u_{e, 1}+\frac{\sigma_{\mathrm{e}}^{l} \alpha}{\sigma_{i}^{l}+\sigma_{\mathrm{e}}^{l}} u_{1}+\frac{\sigma_{\mathrm{e}}^{l} \alpha-\sigma_{i}^{l} \beta}{\sigma_{i}^{l}+\sigma_{\mathrm{e}}^{l}} u_{e, 1} \\
=\boldsymbol{n}_{1}^{T} \frac{\sigma_{\mathrm{e}}^{l} \mathbf{D}_{i}}{\sigma_{i}^{l}+\sigma_{\mathrm{e}}^{l}}\left(\nabla u_{2}+\nabla u_{e, 2}\right)-\boldsymbol{n}_{1}^{T} \frac{\sigma_{i}^{l} \mathbf{D}_{e}}{\sigma_{i}^{l}+\sigma_{\mathrm{e}}^{l}} \nabla u_{e, 2}+\frac{\sigma_{\mathrm{e}}^{l} \alpha}{\sigma_{i}^{l}+\sigma_{\mathrm{e}}^{l}} u_{2}+\frac{\sigma_{\mathrm{e}}^{l} \alpha-\sigma_{i}^{l} \beta}{\sigma_{i}^{l}+\sigma_{\mathrm{e}}^{l}} u_{e, 2}, \\
\boldsymbol{n}_{1}^{T} \mathbf{D}_{i}\left(\nabla u_{1}+\nabla u_{e, 1}\right)+\boldsymbol{n}_{1}^{T} \mathbf{D}_{e} \nabla u_{e, 1}+\alpha u_{1}+(\alpha+\beta) u_{e, 1} \\
=\boldsymbol{n}_{1}^{T} \mathbf{D}_{i}\left(\nabla u_{2}+\nabla u_{e, 2}\right)+\boldsymbol{n}_{1}^{T} \mathbf{D}_{e} \nabla u_{e, 2}+\alpha u_{2}+(\alpha+\beta) u_{e, 2} .
\end{gathered}
$$

For sake of simplicity we take $\beta$ as a function of $\alpha$, in order to have only one parameter to handle on each interface. In what follows we choose $\beta=\frac{\sigma_{e}^{l}}{\sigma_{i}^{l}} \alpha$. Such choice ensures positivity in the coefficients of trace component of the interface condition and simplifies the extracellular potential in the first condition as well. Besides, this is a key ingredient in a model adaptive procedure based on the Bidomain-Monodomain coupling, that is currently under investigation and will be the subject of a forthcoming paper [25].

Remark 4.1. Since the linear combinations providing (4.2) and (4.3) are the same that provide the nonsymmetric formulation, the flux terms in the interface conditions (4.2) and (4.3) naturally appear as boundary terms in the variational formulation, ensuring well-posedeness for the local subproblems in the coupling.

We define

$$
\boldsymbol{\Pi}=\left[\begin{array}{cc}
\frac{\sigma_{\mathrm{e}}^{l}}{\sigma_{i}^{l}+\sigma_{\mathrm{e}}^{l}} & 0 \\
1 & \frac{\sigma_{i}^{l}+\sigma_{\mathrm{e}}^{l}}{\sigma_{i}^{l}}
\end{array}\right],
$$

and a similar argument along $\Gamma_{21}$ leads us to introduce the Optimized Schwarz Algorithm (OSA) as follows.

Given $\boldsymbol{u}_{2}^{0}$ and $\boldsymbol{n}_{1}^{T} \mathbf{D} \nabla \boldsymbol{u}_{2}^{0}$ on $\Gamma_{12}$, solve for $p \geq 0$ until convergence

$$
\begin{array}{ll}
\frac{\chi C_{m}}{\Delta t} \mathbf{E}_{1} \boldsymbol{u}_{1}^{p+1}-\nabla \cdot \mathbf{D} \nabla \boldsymbol{u}_{1}^{p+1}=\boldsymbol{f}_{1} & \text { in } \Omega_{1} \\
\boldsymbol{n}_{1}^{T} \mathbf{D}_{B} \nabla \boldsymbol{u}_{1}^{p+1}=0 & \text { on } \partial \Omega_{1} \cap \partial \Omega \\
\boldsymbol{n}_{1}^{T} \mathbf{D} \nabla \boldsymbol{u}_{1}^{p+1}+\alpha_{1} \boldsymbol{\Pi} \boldsymbol{u}_{1}^{p+1}=\boldsymbol{n}_{1}^{T} \mathbf{D} \nabla \boldsymbol{u}_{2}^{p}+\alpha_{1} \boldsymbol{\Pi} \boldsymbol{u}_{2}^{p} & \text { on } \Gamma_{12} \\
\frac{\chi C_{m}}{\Delta t} \mathbf{E}_{1} \boldsymbol{u}_{2}^{p+1}-\nabla \cdot \mathbf{D} \nabla \boldsymbol{u}_{2}^{p+1}=\boldsymbol{f}_{2} & \text { in } \Omega_{2} \\
\boldsymbol{n}_{2}^{T} \mathbf{D}_{B} \nabla \boldsymbol{u}_{1}^{p+1}=0 & \text { on } \partial \Omega_{2} \cap \partial \Omega \\
\boldsymbol{n}_{2}^{T} \mathbf{D} \nabla \boldsymbol{u}_{2}^{p+1}+\alpha_{2} \boldsymbol{\Pi} \boldsymbol{u}_{2}^{p+1}=\boldsymbol{n}_{2}^{T} \mathbf{D} \nabla \boldsymbol{u}_{1}^{p+1}+\alpha_{2} \boldsymbol{\Pi} \boldsymbol{u}_{1}^{p+1} & \text { on } \Gamma_{21} .
\end{array}
$$

In the present form the Optimized Schwarz Algorithm is sequential, however it can be easily parallelized by choosing $\boldsymbol{u}_{1}^{p}$ in the right hand side of the last equation in (4.4). In this case, an initial set of data $\boldsymbol{u}_{1}^{0}$ and $\boldsymbol{n}_{2}^{T} \mathbf{D} \nabla \boldsymbol{u}_{1}^{0}$ needs to be assigned as well on $\Gamma_{21}$. 


\subsection{Convergence analysis}

We analyze also the convergence properties of the Optimized Schwarz Algorithm via Fourier analysis, and to this aim we consider again the problem on the infinite domain $\Omega=\mathbb{R}^{3}$, decomposed into $\Omega_{1}=(-\infty, L) \times \mathbb{R}^{2}$, and $\Omega_{2}=(0, \infty) \times \mathbb{R}^{2}$, where the interfaces are $\Gamma_{12}=\{L\} \times \mathbb{R}^{2}$ and $\Gamma_{21}=\{0\} \times \mathbb{R}^{2}$, and the overlap size is $L \geq 0$. We set ourselves in the conditions described in Section 3.1. Once again, it is enough to prove convergence to the zero solution in the absence of forcing terms $\left(\boldsymbol{f}_{1}=\boldsymbol{f}_{2}=0\right)$.

Proposition 4.2. For $\alpha_{1}, \alpha_{2} \geq 0$ and $L>0$, the Optimized Schwarz Algorithm (4.4) converges for every initial guess and for every Fourier mode $\boldsymbol{k}>0$. Its reduction factor is independent of the iteration and is given by

$$
\rho_{u}^{\mathrm{OSA}}\left(\boldsymbol{k}, \alpha_{1}, \alpha_{2}, L\right)=\left|\frac{\alpha_{1}-\sigma_{i}^{l} \sqrt{\eta^{+}(\boldsymbol{k})}}{\alpha_{1}+\sigma_{i}^{l} \sqrt{\eta^{+}(\boldsymbol{k})}}\right| \cdot\left|\frac{\alpha_{2}-\sigma_{i}^{l} \sqrt{\eta^{+}(\boldsymbol{k})}}{\alpha_{2}+\sigma_{i}^{l} \sqrt{\eta^{+}(\boldsymbol{k})}}\right| \mathrm{e}^{-2 \sqrt{\eta^{+}(\boldsymbol{k})} L},
$$

for the error in $u$, and by

$$
\rho_{u_{e}}^{\mathrm{OSA}}\left(\boldsymbol{k}, \alpha_{1}, \alpha_{2}, L\right)=\left|\frac{\alpha_{1}-\sigma_{i}^{l} \sqrt{\eta^{-}(\boldsymbol{k})}}{\alpha_{1}+\sigma_{i}^{l} \sqrt{\eta^{-}(\boldsymbol{k})}}\right| \cdot\left|\frac{\alpha_{2}-\sigma_{i}^{l} \sqrt{\eta^{-}(\boldsymbol{k})}}{\alpha_{2}+\sigma_{i}^{l} \sqrt{\eta^{-}(\boldsymbol{k})}}\right| \mathrm{e}^{-2 \sqrt{\eta^{-}(\boldsymbol{k})} L},
$$

for the error in $u_{e}$, where $\eta^{ \pm}(\boldsymbol{k})$ are the eigenvalues of the $2 \times 2$ matrix $A(\boldsymbol{k})$ defined in (3.3).

For $L=0$ the Optimized Schwarz Algorithm (4.4) converges for every initial guess provided $\alpha_{1}, \alpha_{2} \geq 0$, at least one of them strictly positive, and for every Fourier mode $\boldsymbol{k}>0$. The reduction factors are independent of the iteration and are given by $\rho_{u}^{\mathrm{OSA}}\left(\boldsymbol{k}, \alpha_{1}, \alpha_{2}, 0\right)$ and $\rho_{u_{e}}^{\mathrm{OSA}}\left(\boldsymbol{k}, \alpha_{1}, \alpha_{2}, 0\right)$.

Proof. Given $\widehat{\boldsymbol{u}}_{2}^{p}$ on $\{\boldsymbol{k} \in \mathbb{R}, x \geq 0\}$, defining

$$
\boldsymbol{\Sigma}=\left[\begin{array}{ll}
\sigma_{i}^{l} & 0 \\
\sigma_{i}^{l} & \sigma_{i}^{l}+\sigma_{\mathrm{e}}^{l}
\end{array}\right]
$$

the $(p+1)$-th iteration of the OSA solves in the Fourier space

$$
\begin{array}{lr}
\partial_{x x} \widehat{\boldsymbol{u}}_{1}^{p+1}=A(\boldsymbol{k}) \widehat{\boldsymbol{u}}_{1}^{p+1} & \boldsymbol{k} \in \mathbb{R}, x<L \\
\boldsymbol{\Sigma} \partial_{x} \widehat{\boldsymbol{u}}_{1}^{p+1}+\alpha_{1} \frac{1}{\sigma_{i}^{l}} \boldsymbol{\Sigma} \widehat{\boldsymbol{u}}_{1}^{p+1}=\boldsymbol{\Sigma} \partial_{x} \widehat{\boldsymbol{u}}_{2}^{p}+\alpha_{1} \frac{1}{\sigma_{i}^{l}} \boldsymbol{\Sigma} \widehat{\boldsymbol{u}}_{2}^{p} & \boldsymbol{k} \in \mathbb{R}, x=L \\
\partial_{x x} \widehat{\boldsymbol{u}}_{2}^{p+1}=A(\boldsymbol{k}) \widehat{\boldsymbol{u}}_{2}^{p+1} & \boldsymbol{k} \in \mathbb{R}, x>0 \\
-\boldsymbol{\Sigma} \partial_{x} \widehat{\boldsymbol{u}}_{2}^{p+1}+\alpha_{2} \frac{1}{\sigma_{i}^{l}} \boldsymbol{\Sigma} \widehat{\boldsymbol{u}}_{2}^{p+1}=-\boldsymbol{\Sigma} \partial_{x} \widehat{\boldsymbol{u}}_{1}^{p+1}+\alpha_{2} \frac{1}{\sigma_{i}^{l}} \boldsymbol{\Sigma} \widehat{\boldsymbol{u}}_{1}^{p+1} \boldsymbol{k} \in \mathbb{R}, x=0 .
\end{array}
$$

The general solutions in $\Omega_{1}$ and $\Omega_{2}$ at the $(p+1)$-th iteration are given again by (3.9) and (3.10), where the values of $\boldsymbol{\beta}^{p+1}=\left(\beta_{1}^{p+1}, \beta_{2}^{p+1}\right)$ and $\boldsymbol{\gamma}^{p+1}=\left(\gamma_{1}^{p+1}, \gamma_{2}^{p+1}\right)$ are uniquely determined by the boundary conditions on the interfaces $\{x=0\}$ and $\{x=L\}$.

Since $\boldsymbol{\Sigma}$ is invertible, it can be simplified in both interface conditions. Multiplying both sides of the interface conditions by $\sigma_{i}^{l}$, we get from (4.7), (3.9), and (3.10)

$$
\begin{aligned}
& W(\boldsymbol{k})\left[\alpha_{1} \mathbf{I}_{2}+\sigma_{i}^{l} \mathrm{H}^{1 / 2}(\boldsymbol{k})\right] \boldsymbol{\beta}^{p+1}=W(\boldsymbol{k})\left[\alpha_{1} \mathbf{I}_{2}-\sigma_{i}^{l} \mathrm{H}^{1 / 2}(\boldsymbol{k})\right] \Lambda(\boldsymbol{k}, L) \boldsymbol{\gamma}^{p} \\
& W(\boldsymbol{k})\left[\alpha_{2} \mathbf{I}_{2}+\sigma_{i}^{l} \mathrm{H}^{1 / 2}(\boldsymbol{k})\right] \boldsymbol{\gamma}^{p+1}=W(\boldsymbol{k})\left[\alpha_{2} \mathbf{I}_{2}-\sigma_{i}^{l} \mathrm{H}^{1 / 2}(\boldsymbol{k})\right] \Lambda(\boldsymbol{k}, L) \boldsymbol{\beta}^{p+1},
\end{aligned}
$$

where $W(\boldsymbol{k})$ and $\mathrm{H}(\boldsymbol{k})$ are defined in (3.7), while $\Lambda(\boldsymbol{k}, L)$ has been defined in (3.11). 
For $\boldsymbol{k}>0$ the matrices $W(\boldsymbol{k})$ and $\mathrm{H}^{1 / 2}(\boldsymbol{k})$ are invertible. Moreover, since both $\mathrm{H}^{1 / 2}(\boldsymbol{k})$ and $\Lambda(\boldsymbol{k}, L)$ are diagonal, we have from (4.8)

$$
\begin{aligned}
\boldsymbol{\gamma}^{p+1} & =\left[\alpha_{2} \mathbf{I}_{2}+\sigma_{i}^{l} \mathrm{H}^{1 / 2}(\boldsymbol{k})\right]^{-1}\left[\alpha_{2} \mathbf{I}_{2}-\sigma_{i}^{l} \mathrm{H}^{1 / 2}(\boldsymbol{k})\right]\left[\alpha_{1} \mathbf{I}_{2}+\sigma_{i}^{l} \mathrm{H}^{1 / 2}(\boldsymbol{k})\right]^{-1}\left[\alpha_{1} \mathbf{I}_{2}-\sigma_{i}^{l} \mathrm{H}^{1 / 2}(\boldsymbol{k})\right] \Lambda^{2}(\boldsymbol{k}, L) \boldsymbol{\gamma}^{p} \\
& =S\left(\boldsymbol{k}, \alpha_{1}, \alpha_{2}\right) \Lambda^{2}(\boldsymbol{k}) \boldsymbol{\gamma}^{p}(\boldsymbol{k}),
\end{aligned}
$$

so that, given $\gamma^{0}(\boldsymbol{k})$,

$$
\boldsymbol{\gamma}^{p}(\boldsymbol{k})=\left[S\left(\boldsymbol{k}, \alpha_{1}, \alpha_{2}\right)\right]^{p} \Lambda^{2 p}(\boldsymbol{k}, L) \boldsymbol{\gamma}^{0}(\boldsymbol{k}) .
$$

The reduction factor of the Schwarz Algorithm is then given by the spectral radius of the matrix product $S\left(\boldsymbol{k}, \alpha_{1}, \alpha_{2}\right) \Lambda^{2}(\boldsymbol{k}, L)$, and it is thus independent of the iteration $p$. Moreover, since for any Fourier mode $\boldsymbol{k}>0$,

$$
S\left(\boldsymbol{k}, \alpha_{1}, \alpha_{2}\right)=\left[\begin{array}{ccc}
\frac{\alpha_{1}-\sigma_{i}^{l} \sqrt{\eta^{+}(\boldsymbol{k})}}{\alpha_{1}+\sigma_{i}^{l} \sqrt{\eta^{+}(\boldsymbol{k})}} \cdot \frac{\alpha_{2}-\sigma_{i}^{l} \sqrt{\eta^{+}(\boldsymbol{k})}}{\alpha_{2}+\sigma_{i}^{l} \sqrt{\eta^{+}(\boldsymbol{k})}} & 0 \\
0 & \frac{\alpha_{1}-\sigma_{i}^{l} \sqrt{\eta^{-}(\boldsymbol{k})}}{\alpha_{1}+\sigma_{i}^{l} \sqrt{\eta^{-}(\boldsymbol{k})}} \cdot \frac{\alpha_{2}-\sigma_{i}^{l} \sqrt{\eta^{-}(\boldsymbol{k})}}{\alpha_{2}+\sigma_{i}^{l} \sqrt{\eta^{-}(\boldsymbol{k})}}
\end{array}\right],
$$

the reduction factors of the Optimized Schwarz algorithm are given by (4.5) and (4.6).

To conclude the proof, observe that for $L=0, \Lambda(\boldsymbol{k}, 0)=\mathbf{I}_{2}$, the iteration matrix reduces to $S\left(\boldsymbol{k}, \alpha_{1}, \alpha_{2}\right)$ and, for any $\alpha_{1}, \alpha_{2} \geq 0$ we have

$$
\left|\frac{\alpha_{1}-\sigma_{i}^{l} \sqrt{\eta^{+}(\boldsymbol{k})}}{\alpha_{1}+\sigma_{i}^{l} \sqrt{\eta^{+}(\boldsymbol{k})}}\right| \cdot\left|\frac{\alpha_{2}-\sigma_{i}^{l} \sqrt{\eta^{+}(\boldsymbol{k})}}{\alpha_{2}+\sigma_{i}^{l} \sqrt{\eta^{+}(\boldsymbol{k})}}\right|<1 \quad\left|\frac{\alpha_{1}-\sigma_{i}^{l} \sqrt{\eta^{-}(\boldsymbol{k})}}{\alpha_{1}+\sigma_{i}^{l} \sqrt{\eta^{-}(\boldsymbol{k})}}\right| \cdot\left|\frac{\alpha_{2}-\sigma_{i}^{l} \sqrt{\eta^{-}(\boldsymbol{k})}}{\alpha_{2}+\sigma_{i}^{l} \sqrt{\eta^{-}(\boldsymbol{k})}}\right|<1,
$$

for $\boldsymbol{k}>0$, provided at least one among $\alpha_{1}$ and $\alpha_{2}$ is strictly positive.

Owing to (4.10), the following corollary holds.

Corollary 4.3. For any $\alpha_{1}, \alpha_{2} \geq 0$, at least one of which strictly positive, we have for all $\boldsymbol{k}>0$,

$$
\rho_{u}^{\mathrm{OSA}}\left(\boldsymbol{k}, \alpha_{1}, \alpha_{2}, L\right)<\rho_{u}^{\mathrm{Cla}}(\boldsymbol{k}, L) \quad \rho_{u_{e}}^{\mathrm{OSA}}\left(\boldsymbol{k}, \alpha_{1}, \alpha_{2}, L\right)<\rho_{u_{e}}^{\mathrm{Cla}}(\boldsymbol{k}, L) .
$$

We point out that starting the algorithm by solving within $\Omega_{2}$ first would provide the same result, since again one gets $\boldsymbol{\beta}^{p+1}=S\left(\boldsymbol{k}, \alpha_{1}, \alpha_{2}\right) \Lambda^{2}(\boldsymbol{k}, L) \boldsymbol{\beta}^{p}$.

Remark 4.4. Differently from the classical Schwarz Algorithm, the Optimized Schwarz converges also in the absence of overlap for all Fourier mode $\boldsymbol{k}>0$. Again, since $\boldsymbol{\eta}^{-}(0)=0, \rho_{u_{e}}^{\mathrm{OSA}}\left(0, \alpha_{1}, \alpha_{2}, L\right)=1$ regardless of the overlap size $L$ and the parameters $\alpha_{1}$ and $\alpha_{2}$, thus the Optimized Schwarz algorithm does not converge in its iterative form for the frequency $\boldsymbol{k}=0$. This problem can be accounted for in the same way outlined in the case of the Classical Schwarz algorithm.

In the next sections we focus on the choice of the parameters $\alpha_{l}(l=1,2)$ in the Robin interface conditions. Since $\Lambda(\boldsymbol{k}, L)$ does not depend on neither $\alpha_{1}$ nor $\alpha_{2}$, the optimal parameters can be sought in the nonoverlapping case $L=0$. Ideally, the optimal parameters force the convergence factors $\rho_{u}^{\mathrm{OSA}}\left(\boldsymbol{k}, \alpha_{1}, \alpha_{2}, L\right)$ and $\rho_{u_{e}}^{\mathrm{OSA}}\left(\boldsymbol{k}, \alpha_{1}, \alpha_{2}, L\right)$ to be identically zero, so that convergence is attained in a number of iterations equal to the number of subdomains (two, in the case at hand).

\subsection{One-sided Robin interface conditions}

The classical and simplest approach in the Optimized Schwarz literature consists in choosing the same Robin parameter on both sides of the interface [34]. Such conditions are referred to as one-sided Robin interface conditions. 
If we choose $\alpha_{1}=\alpha_{2}=\alpha$, the iteration matrix is given by

$$
S(\boldsymbol{k}, \alpha)=\left(\left[\alpha \mathbf{I}_{2}+\sigma_{i}^{l} \mathrm{H}^{1 / 2}(\boldsymbol{k})\right]^{-1}\left[\alpha \mathbf{I}_{2}-\sigma_{i}^{l} \mathrm{H}^{1 / 2}(\boldsymbol{k})\right]\right)^{2}=\left[\begin{array}{cc}
\left|\frac{\alpha-\sigma_{i}^{l} \sqrt{\eta^{+}(\boldsymbol{k})}}{\alpha+\sigma_{i}^{l} \sqrt{\eta^{+}(\boldsymbol{k})}}\right|^{2} & 0 \\
0 & \left|\frac{\alpha-\sigma_{i}^{l} \sqrt{\eta^{-}(\boldsymbol{k})}}{\alpha+\sigma_{i}^{l} \sqrt{\eta^{-}(\boldsymbol{k})}}\right|^{2}
\end{array}\right],
$$

thus we have

$$
\rho_{u}^{\mathrm{OSA}}(\boldsymbol{k}, \alpha)=\left|\frac{\alpha-\sigma_{i}^{l} \sqrt{\eta^{+}(\boldsymbol{k})}}{\alpha+\sigma_{i}^{l} \sqrt{\eta^{+}(\boldsymbol{k})}}\right|^{2}, \quad \rho_{u_{e}}^{\mathrm{OSA}}(\boldsymbol{k}, \alpha)=\left|\frac{\alpha-\sigma_{i}^{l} \sqrt{\eta^{-}(\boldsymbol{k})}}{\alpha+\sigma_{i}^{l} \sqrt{\eta^{-}(\boldsymbol{k})}}\right|^{2} .
$$

In [28] the iterative mapping is scalar and the optimal parameter can be easily identified. Here the iterative mapping is a matrix: it is diagonal but, differently from what happens, for instance, for Maxwell's equations (see [3]), it is not a multiple of the identity, thus it is not possible to devise a single parameter (even depending on the tangential frequency $\boldsymbol{k})$ that both $\rho_{u}^{\mathrm{OSA}}(\boldsymbol{k}, \alpha)$ and $\rho_{u_{e}}^{\mathrm{OSA}}(\boldsymbol{k}, \alpha)$ vanish identically. Indeed, only one of the two eigenvalues can be annihilated at a given time.

The standard approach in Optimized Schwarz literature consists in optimizing the parameter $\alpha$ by minimizing the convergence rate over all the relevant frequencies of the problem: such approach amounts to solve the minmax problem

$$
\min _{\alpha \in \mathbb{R}^{+}} \max _{\boldsymbol{k} \in\left[\boldsymbol{k}_{\min }, \boldsymbol{k}_{\max }\right]}\left(\rho_{u}^{\mathrm{OSA}}(\boldsymbol{k}, \alpha), \rho_{u_{e}}^{\mathrm{OSA}}(\boldsymbol{k}, \alpha)\right),
$$

where $\boldsymbol{k}_{\text {min }}$ is the smallest frequency relevant to the problem and $\boldsymbol{k}_{\max }$ is the largest frequency supported by the numerical grid, which is of the order $\pi / h$, being $h$ the mesh size [20,24]. By an equioscillation principle, one is led to seek for the solution of the min-max problem as $\alpha^{\mathrm{opt}}$ such that $\rho\left(\boldsymbol{k}_{\min }, \alpha^{\mathrm{opt}}\right)=\rho\left(\boldsymbol{k}_{\max }, \alpha^{\mathrm{opt}}\right)$, namely

$$
\alpha^{\mathrm{opt}}=\sigma_{i}^{l}\left(\eta^{-}\left(\boldsymbol{k}_{\min }\right) \eta^{+}\left(\boldsymbol{k}_{\max }\right)\right)^{1 / 4}
$$

Unfortunately, although effective to optimize the interface Robin parameter in several situations (see, e.g. $[3,16,20,24])$ this would not be a good choice in the case at hand. Infact, $\lim _{\boldsymbol{k} \rightarrow \infty} \rho_{u}^{\mathrm{OSA}}(\boldsymbol{k}, \alpha)=1$, and since $\rho_{u_{e}}^{\mathrm{OSA}}(\mathbf{0}, \alpha)=1$ for all $\alpha$, also $\rho_{u_{e}}^{\mathrm{OSA}}\left(\boldsymbol{k}_{\mathrm{min}}, \alpha\right) \sim 1$, the overall convergence rate would be very close to 1 . We thus consider different possible choices of the parameter $\alpha$, based on zero-th order Taylor expansions of $\eta^{+}(\boldsymbol{k})$ and $\eta^{-}(\boldsymbol{k})$ around different values of the frequency $\boldsymbol{k}$, that will be used in the numerical tests of Section 6 .

- O1: $\quad \alpha=\sigma_{i}^{l} \sqrt{\boldsymbol{\eta}^{+}(\mathbf{0})}$.

The interface condition is based on the Taylor expansion of $\sqrt{\boldsymbol{\eta}^{+}(\boldsymbol{k})}$ around $\mathbf{0}$. It damps the zero frequency in $\rho_{u}^{\mathrm{OSA}}(\boldsymbol{k}, \alpha)$, while no frequency is damped in $\rho_{u_{e}}^{\mathrm{OSA}}(\boldsymbol{k}, \alpha)$.

- O2: $\quad \alpha=\sigma_{i}^{l} \sqrt{\boldsymbol{\eta}^{-}\left(h \boldsymbol{k}_{\max }\right)}$.

The interface condition is based on the Taylor expansion of $\sqrt{\boldsymbol{\eta}^{-}(\boldsymbol{k})}$ around $h \boldsymbol{k}_{\max }$, which can be regarded as an approximation of $\boldsymbol{k}_{\text {min. }}$. It damps the frequency $h \boldsymbol{k}_{\text {max }}$ in $\rho_{u_{e}}^{\text {OSA }}(\boldsymbol{k}, \alpha)$, while no frequency is damped in $\rho_{u}^{\mathrm{OSA}}(\boldsymbol{k}, \alpha)$.

- O3: $\quad \alpha=\sigma_{i}^{l} \sqrt{\boldsymbol{\eta}^{-}\left(\boldsymbol{k}_{\max } / 2\right)}$.

The interface condition is based on the Taylor expansion of $\sqrt{\boldsymbol{\eta}^{-}(\boldsymbol{k})}$ around $\boldsymbol{k}_{\max } / 2$. It damps the frequency $\boldsymbol{k}_{\max } / 2$ in $\rho_{u_{e}}^{\mathrm{OSA}}(\boldsymbol{k}, \alpha)$, while no frequency is damped in $\rho_{u}^{\mathrm{OSA}}(\boldsymbol{k}, \alpha)$.

- O4: $\quad \alpha=\sigma_{i}^{l} \sqrt{\boldsymbol{\eta}^{-}\left(\boldsymbol{k}_{\max }\right)}$.

The interface condition is based on the Taylor expansion of $\sqrt{\boldsymbol{\eta}^{-}(\boldsymbol{k})}$ around the maximal frequency $\boldsymbol{k}_{\max }$. It damps the frequency $\boldsymbol{k}_{\max }$ in $\rho_{u_{e}}^{\mathrm{OSA}}(\boldsymbol{k}, \alpha)$, while no frequency is damped in $\rho_{u}^{\mathrm{OSA}}(\boldsymbol{k}, \alpha)$. 

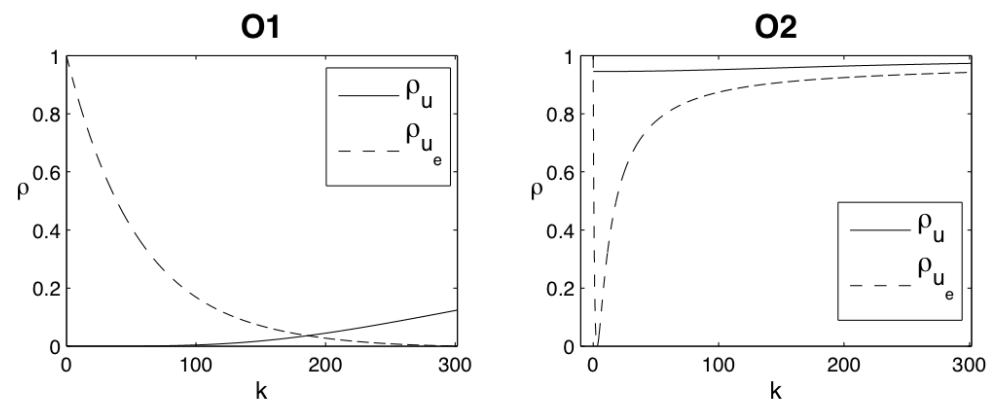

03
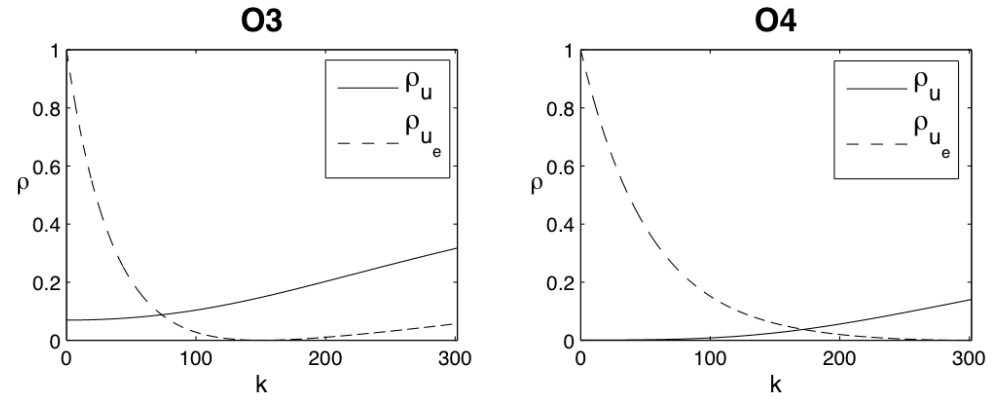

FIgURE 3. Reduction factors $\rho_{u}^{\mathrm{OSA}}$ and $\rho_{u_{e}}^{\mathrm{OSA}}$ as a function of the frequency $\boldsymbol{k}$ with nonoverlapping decompositions: one-sided conditions.

We plot in Figure 3 the convergence rates, as a function of the frequency variable $\boldsymbol{k}$, of the one-sided Robin condition: the solid line refers to the convergence rate $\rho_{u}^{\mathrm{OSA}}$, the dashed line to the convergence rate $\rho_{u_{e}}^{\mathrm{OSA}}$. We choose $\boldsymbol{k}_{\max }=\pi / h$, thus $\boldsymbol{k}_{\min }=\pi$. From Figure 3, we expect in general a faster convergence of the $u$ variable with respect to the $u_{e}$ variable, except in the case of the interface condition O2. Moreover, we expect the interface condition $\mathbf{O} 2$ to behave very poorly in the case of a non-overlapping decomposition.

\subsection{Two-sided Robin interface conditions}

An improvement can be obtained by the use of two-sided interface conditions (using different parameters on the two sides of the interface, see [20,22] for an application to Helmholtz problem), which proved to enhance the convergence rate of the Optimized Schwarz Algorithm. In this case the iteration matrix is $M\left(\boldsymbol{k}, \alpha_{1}, \alpha_{2}, L\right)=$ $S\left(\boldsymbol{k}, \alpha_{1}, \alpha_{2}\right) \Lambda^{2}(\boldsymbol{k}, L)$, where $S\left(\boldsymbol{k}, \alpha_{1}, \alpha_{2}\right)$ is the one in (4.9), and the optimal parameters are given by

$$
\alpha_{1}^{\text {exact }}(\boldsymbol{k})=\sigma_{i}^{l} \sqrt{\eta^{+}(\boldsymbol{k})} \quad \alpha_{2}^{\text {exact }}(\boldsymbol{k})=\sigma_{i}^{l} \sqrt{\eta^{-}(\boldsymbol{k})} .
$$

In fact, $\alpha_{1}^{\text {exact }}(\boldsymbol{k})$ and $\alpha_{2}^{\text {exact }}(\boldsymbol{k})$ are actually the symbols of two linear, pseudodifferential, operators acting along the interface. These expressions are not viable since their back transform in the space of physical variables is a nonlocal operator. We resort therefore to a different, even suboptimal, choice, and we approximate $\alpha_{1}^{\text {exact }}(\boldsymbol{k})$ and $\alpha_{2}^{\text {exact }}(\boldsymbol{k})$ with constants, by means of their zero-th order Taylor expansion as done in the previous section. We obtain the following two-sided interface conditions, that will be used in the numerical tests of Section 6 .

$$
\text { - T1: } \quad \alpha_{1}=\sigma_{i}^{l} \sqrt{\boldsymbol{\eta}^{+}(\mathbf{0})}, \quad \alpha_{2}=\sigma_{i}^{l} \sqrt{\boldsymbol{\eta}^{-}\left(h \boldsymbol{k}_{\max }\right)}
$$

The interface condition is based on the Taylor expansion of $\sqrt{\boldsymbol{\eta}^{+}(\boldsymbol{k})}$ around $\mathbf{0}$ and of $\sqrt{\eta^{-}(\boldsymbol{k})}$ around $h \boldsymbol{k}_{\max }$. It damps the zero frequency in $\rho_{u}^{\mathrm{OSA}}\left(\boldsymbol{k}, \alpha_{1}, \alpha_{2}\right)$, and the frequency $h \boldsymbol{k}_{\max }$ in $\rho_{u_{e}}^{\mathrm{OSA}}\left(\boldsymbol{k}, \alpha_{1}, \alpha_{2}\right)$.

$$
\text { - T2: } \quad \alpha_{1}=\sigma_{i}^{l} \sqrt{\boldsymbol{\eta}^{+}(\mathbf{0})}, \quad \alpha_{2}=\sigma_{i}^{l} \sqrt{\boldsymbol{\eta}^{-}\left(\boldsymbol{k}_{\max } / 2\right)}
$$

The interface condition is based on the Taylor expansion of $\sqrt{\boldsymbol{\eta}^{+}(\boldsymbol{k})}$ around $\mathbf{0}$ and of $\sqrt{\eta^{-}(\boldsymbol{k})}$ around $\boldsymbol{k}_{\max } / 2$. It damps the zero frequency in $\rho_{u}^{\mathrm{OSA}}\left(\boldsymbol{k}, \alpha_{1}, \alpha_{2}\right)$, and the frequency $\boldsymbol{k}_{\max } / 2$ in $\rho_{u_{e}}^{\mathrm{OSA}}\left(\boldsymbol{k}, \alpha_{1}, \alpha_{2}\right)$. 
T1

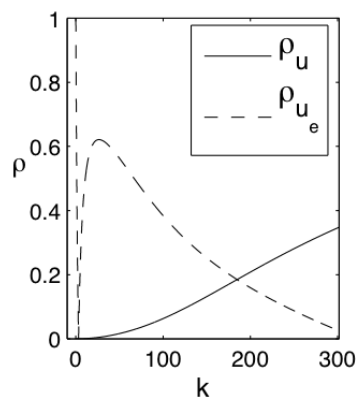

T4

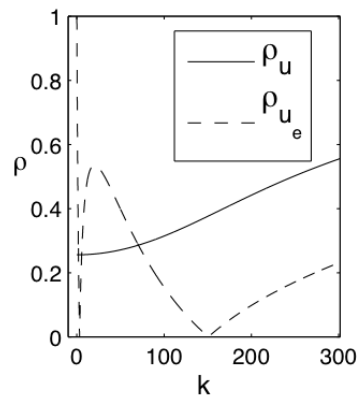

T2

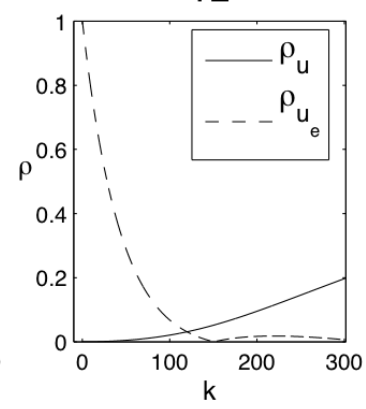

T5

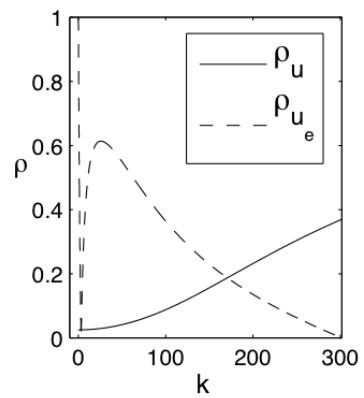

T3

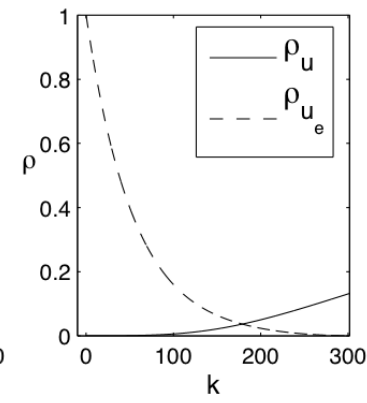

T6

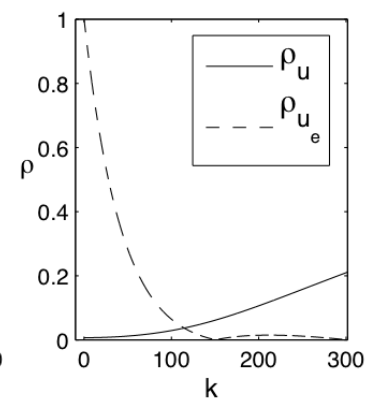

FIgURE 4. Reduction factors $\rho_{u}^{\mathrm{OSA}}$ and $\rho_{u_{e}}^{\mathrm{OSA}}$ as a function of the frequency $\boldsymbol{k}$ with nonoverlapping decompositions: two-sided conditions.

- T3: $\quad \alpha_{1}=\sigma_{i}^{l} \sqrt{\boldsymbol{\eta}^{+}(\mathbf{0})}, \quad \alpha_{2}=\sigma_{i}^{l} \sqrt{\boldsymbol{\eta}^{-}\left(\boldsymbol{k}_{\max }\right)}$

The interface condition is based on the Taylor expansion of $\sqrt{\boldsymbol{\eta}^{+}(\boldsymbol{k})}$ around $\mathbf{0}$ and of $\sqrt{\eta^{-}(\boldsymbol{k})}$ around $\boldsymbol{k}_{\max }$. It damps the zero frequency in $\rho_{u}^{\mathrm{OSA}}\left(\boldsymbol{k}, \alpha_{1}, \alpha_{2}\right)$, and the frequency $\boldsymbol{k}_{\max }$ in $\rho_{u_{e}}^{\mathrm{OSA}}\left(\boldsymbol{k}, \alpha_{1}, \alpha_{2}\right)$.

- T4: $\quad \alpha_{1}=\sigma_{i}^{l} \sqrt{\boldsymbol{\eta}^{-}\left(h \boldsymbol{k}_{\max }\right)}, \quad \alpha_{2}=\sigma_{i}^{l} \sqrt{\boldsymbol{\eta}^{-}\left(\boldsymbol{k}_{\max } / 2\right)}$

The interface condition is based on the Taylor expansion of $\sqrt{\eta^{-}(\boldsymbol{k})}$ around $h \boldsymbol{k}_{\max }$ and $\boldsymbol{k}_{\max } / 2$. It damps the frequencies $h \boldsymbol{k}_{\max }$ and $\boldsymbol{k}_{\max } / 2$ in $\rho_{u_{e}}^{\mathrm{OSA}}\left(\boldsymbol{k}, \alpha_{1}, \alpha_{2}\right)$, while no frequency is damped in $\rho_{u}^{\mathrm{OSA}}\left(\boldsymbol{k}, \alpha_{1}, \alpha_{2}\right)$.

- T5: $\quad \alpha_{1}=\sigma_{i}^{l} \sqrt{\boldsymbol{\eta}^{-}\left(h \boldsymbol{k}_{\max }\right)}, \quad \alpha_{2}=\sigma_{i}^{l} \sqrt{\boldsymbol{\eta}^{-}\left(\boldsymbol{k}_{\max }\right)}$

The interface condition is based on the Taylor expansion of $\sqrt{\eta^{-}(\boldsymbol{k})}$ around $h \boldsymbol{k}_{\max }$ and $\boldsymbol{k}_{\max }$. It damps the frequencies $h \boldsymbol{k}_{\text {max }}$ and $\boldsymbol{k}_{\max }$ in $\rho_{u_{e}}^{\mathrm{OSA}}\left(\boldsymbol{k}, \alpha_{1}, \alpha_{2}\right)$, while no frequency is damped in $\rho_{u}^{\mathrm{OSA}}\left(\boldsymbol{k}, \alpha_{1}, \alpha_{2}\right)$.

- T6: $\quad \alpha_{1}=\sigma_{i}^{l} \sqrt{\boldsymbol{\eta}^{-}\left(\boldsymbol{k}_{\max } / 2\right)}, \quad \alpha_{2}=\sigma_{i}^{l} \sqrt{\boldsymbol{\eta}^{-}\left(\boldsymbol{k}_{\max }\right)}$

The interface condition is based on the Taylor expansion of $\sqrt{\eta^{-}(\boldsymbol{k})}$ around $\boldsymbol{k}_{\max } / 2$ and $\boldsymbol{k}_{\max }$. It damps the frequencies $\boldsymbol{k}_{\max } / 2$ and $\boldsymbol{k}_{\max }$ in $\rho_{u_{e}}^{\mathrm{OSA}}\left(\boldsymbol{k}, \alpha_{1}, \alpha_{2}\right)$, while no frequency is damped in $\rho_{u}^{\mathrm{OSA}}\left(\boldsymbol{k}, \alpha_{1}, \alpha_{2}\right)$.

We plot in Figure 4 the convergence rates, as a function of the frequency variable $\boldsymbol{k}$, of the two-sided Robin conditions: the solid line refers to the convergence rate $\rho_{u}^{\mathrm{OSA}}$, the dashed line to the convergence rate $\rho_{u_{e}}^{\mathrm{OSA}}$. Again, we choose $\boldsymbol{k}_{\max }=\pi / h$, and $\boldsymbol{k}_{\min }=\pi$. From Figure 4 , we expect in general a faster convergence of the $u$ variable with respect to the $u_{e}$ variable.

\section{Optimized Schwarz Method as A Preconditioner}

In this section we focus on the discrete version of the Optimized Schwarz Algorithms. We consider the case in which the subdomains $\Omega_{1}$ and $\Omega_{2}$ are obtained from a nonoverlapping partitioning of a regular triangulation 
of the whole computational domain $\Omega$, in order to have matching meshes on the interface. We let $\mathbf{B}_{l}$ denote the matrix defined in (2.7) when computed on a triangulation of $\Omega_{l}(l=1,2)$. We denote by $R_{l, \Gamma}(l=1,2)$ the restriction matrices from $\Omega_{l}$ to $\Gamma$, respectively, and we let

$$
\boldsymbol{R}_{l, \Gamma}=\left[\begin{array}{ll}
R_{l, \Gamma} & 0 \\
0 & R_{l, \Gamma}
\end{array}\right]
$$

be its extension to pairs $\boldsymbol{u}_{l}=\left(u_{l}, u_{e, l}\right)^{T}$.

By introducing, at the space continuous level, the interface variables on $\Gamma$

$$
\boldsymbol{\lambda}_{1}^{p}=\boldsymbol{n}_{1}^{T} \mathbf{D} \nabla \boldsymbol{u}_{2}^{p}+\alpha_{1} \boldsymbol{\Pi} \boldsymbol{u}_{2}^{p} \quad \boldsymbol{\lambda}_{2}^{p}=\boldsymbol{n}_{2}^{T} \mathbf{D} \nabla \boldsymbol{u}_{1}^{p}+\alpha_{2} \boldsymbol{\Pi} \boldsymbol{u}_{1}^{p},
$$

the parallel version of the Optimized Schwarz Algorithm reads

$$
\begin{aligned}
& \begin{cases}\frac{\chi C_{m}}{\Delta t} \mathbf{E}_{1} \boldsymbol{u}_{1}^{p+1}-\nabla \cdot \mathbf{D} \nabla \boldsymbol{u}_{1}^{p+1}=\boldsymbol{f}_{1} & \text { in } \Omega_{1} \\
\boldsymbol{n}_{1}^{T} \mathbf{D}_{B} \nabla \boldsymbol{u}_{1}^{p+1}=0 & \text { on } \partial \Omega_{1} \cap \partial \Omega \\
\boldsymbol{n}_{1}^{T} \mathbf{D} \nabla \boldsymbol{u}_{1}^{p+1}+\alpha_{1} \boldsymbol{\Pi} \boldsymbol{u}_{1}^{p+1}=\boldsymbol{\lambda}_{1}^{p} & \text { on } \Gamma\end{cases} \\
& \begin{cases}\frac{\chi C_{m}}{\Delta t} \mathbf{E}_{1} \boldsymbol{u}_{2}^{p+1}-\nabla \cdot \mathbf{D} \nabla \boldsymbol{u}_{2}^{p+1}=\boldsymbol{f}_{2} & \text { in } \Omega_{2} \\
\boldsymbol{n}_{2}^{T} \mathbf{D}_{B} \nabla \boldsymbol{u}_{1}^{p+1}=0 & \text { on } \partial \Omega_{2} \cap \partial \Omega \\
\boldsymbol{n}_{2}^{T} \mathbf{D} \nabla \boldsymbol{u}_{2}^{p+1}+\alpha_{2} \boldsymbol{\Pi} \boldsymbol{u}_{2}^{p+1}=\boldsymbol{\lambda}_{2}^{p} & \text { on } \Gamma\end{cases} \\
& \boldsymbol{\lambda}_{2}^{p+1}=\boldsymbol{n}_{2}^{T} \mathbf{D} \nabla \boldsymbol{u}_{1}^{p+1}+\alpha_{2} \boldsymbol{\Pi} \boldsymbol{u}_{1}^{p+1} \quad \text { on } \Gamma \\
& \boldsymbol{\lambda}_{1}^{p+1}=\boldsymbol{n}_{1}^{T} \mathbf{D} \nabla \boldsymbol{u}_{2}^{p+1}+\alpha_{1} \boldsymbol{\Pi} \boldsymbol{u}_{2}^{p+1} \quad \text { on } \Gamma .
\end{aligned}
$$

With a little abuse of notation, we denote by $\boldsymbol{u}_{l}^{p}, \boldsymbol{\lambda}_{l}^{p}$, and $\boldsymbol{f}_{l}(l=1,2)$ the discrete version of the unknowns and the forcing terms. We denote by $M_{\Gamma}$ the mass matrix on the interface $\Gamma$, and we set

$$
R_{1}=R_{1, \Gamma}^{T} M_{\Gamma} R_{1, \Gamma} \quad R_{2}=R_{2, \Gamma}^{T} M_{\Gamma} R_{2, \Gamma} .
$$

We let, for $l=1,2$,

$$
\mathbf{A}_{l}=\mathbf{B}_{l}+\alpha_{l}^{*} \boldsymbol{\Pi}_{l}
$$

where

$$
\boldsymbol{\Pi}_{1}=\left[\begin{array}{ll}
\frac{\sigma_{\mathrm{e}}^{l}}{\sigma_{i}^{l}+\sigma_{\mathrm{e}}^{l}} R_{1} & \mathbf{0} \\
R_{1} & \frac{\sigma_{i}^{l}+\sigma_{\mathrm{e}}^{l}}{\sigma_{i}^{l}} R_{1}
\end{array}\right] \quad \boldsymbol{\Pi}_{2}=\left[\begin{array}{ll}
\frac{\sigma_{\mathrm{e}}^{l}}{\sigma_{i}^{l}+\sigma_{\mathrm{e}}^{l}} R_{2} & \mathbf{0} \\
R_{2} & \frac{\sigma_{i}^{l}+\sigma_{\mathrm{e}}^{l}}{\sigma_{i}^{l}} R_{2}
\end{array}\right] .
$$

With these positions, the discrete version of (5.1) reads

$$
\begin{gathered}
\mathbf{A}_{1} \boldsymbol{u}_{1}^{p+1}=\boldsymbol{f}_{1}+\mathbf{R}_{1, \Gamma}^{T} \boldsymbol{\lambda}_{1}^{p} \quad \mathbf{A}_{2} \boldsymbol{u}_{2}^{p+1}=\boldsymbol{f}_{2}+\mathbf{R}_{2, \Gamma}^{T} \boldsymbol{\lambda}_{2}^{p} \\
\boldsymbol{\lambda}_{1}^{p+1}=\mathbf{R}_{2, \Gamma}\left(\boldsymbol{f}_{2}-\mathbf{B}_{2} \boldsymbol{u}_{2}^{p+1}\right)+\alpha_{1}^{*} \mathbf{R}_{2, \Gamma} \boldsymbol{\Pi}_{2} \boldsymbol{u}_{2}^{p+1} \\
\boldsymbol{\lambda}_{2}^{p+1}=\mathbf{R}_{1, \Gamma}\left(\boldsymbol{f}_{1}-\mathbf{B}_{1} \boldsymbol{u}_{1}^{p+1}\right)+\alpha_{2}^{*} \mathbf{R}_{1, \Gamma} \boldsymbol{\Pi}_{1} \boldsymbol{u}_{1}^{p+1}
\end{gathered}
$$

which, owing to (5.2), becomes

$$
\begin{aligned}
& \boldsymbol{\lambda}_{1}^{p+1}=-\boldsymbol{\lambda}_{2}^{p}+\left(\alpha_{1}^{*}+\alpha_{2}^{*}\right) \mathbf{R}_{2, \Gamma} \boldsymbol{\Pi}_{2}\left(\mathbf{A}_{2}^{-1} \boldsymbol{f}_{2}+\mathbf{A}_{2}^{-1} \mathbf{R}_{2, \Gamma}^{T} \boldsymbol{\lambda}_{2}^{p}\right) \\
& \boldsymbol{\lambda}_{2}^{p+1}=-\boldsymbol{\lambda}_{1}^{p}+\left(\alpha_{1}^{*}+\alpha_{2}^{*}\right) \mathbf{R}_{1, \Gamma} \boldsymbol{\Pi}_{1}\left(\mathbf{A}_{1}^{-1} \boldsymbol{f}_{1}+\mathbf{A}_{1}^{-1} \mathbf{R}_{1, \Gamma}^{T} \boldsymbol{\lambda}_{1}^{p}\right) .
\end{aligned}
$$


The above scheme is a fixed point iteration procedure to solve system

$$
\left[\begin{array}{cc}
\mathbf{I} & \mathbf{I}-\left(\alpha_{1}^{*}+\alpha_{2}^{*}\right) \boldsymbol{\Sigma}_{2} \\
\mathbf{I}-\left(\alpha_{1}^{*}+\alpha_{2}^{*}\right) \boldsymbol{\Sigma}_{1} & \mathbf{I}
\end{array}\right]\left[\begin{array}{c}
\boldsymbol{\lambda}_{1} \\
\boldsymbol{\lambda}_{2}
\end{array}\right]=\left(\alpha_{1}^{*}+\alpha_{2}^{*}\right)\left[\begin{array}{l}
\mathbf{R}_{2, \Gamma} \boldsymbol{\Pi}_{2} \mathbf{A}_{2}^{-1} \boldsymbol{f}_{2} \\
\mathbf{R}_{1, \Gamma} \boldsymbol{\Pi}_{1} \mathbf{A}_{1}^{-1} \boldsymbol{f}_{1}
\end{array}\right],
$$

where

$$
\boldsymbol{\Sigma}_{1}=\mathbf{R}_{1, \Gamma} \boldsymbol{\Pi}_{1} \mathbf{A}_{1}^{-1} \mathbf{R}_{1, \Gamma}^{T} \quad \boldsymbol{\Sigma}_{2}=\mathbf{R}_{2, \Gamma} \boldsymbol{\Pi}_{2} \mathbf{A}_{2}^{-1} \mathbf{R}_{2, \Gamma}^{T} .
$$

We solve systems (5.5) above with a Krylov subspace method. This corresponds to using the Optimized Schwarz methods as preconditioners. Notice that, for each iteration of Krylov method we have to solve (5.4), whose computationl cost is mainly due to the solution of a system associated to $A_{l}$ in each subdomain. Therefore the computational cost of a Krylov iteration is comparable to the cost of a OSA iteration. However, Krylov method requires the additional computational cost for determining the right-hand side of system (5.5), and, once the converged values of $\boldsymbol{\lambda}_{1,2}$ have been obtained, to determine the final values of $\boldsymbol{u}_{1,2}$, using $(5.3)_{1}$. It follows that the cost of solving system 5.5 in $n$ iterations can be extimated with the cost of solving $n+2$ OSA iterations.

\section{Numerical Results}

Numerical tests presented in this section have two different goals. We first focus on the stationary problem to be solved within each time step. At this level we test the effectiveness and the robustness of the Optimized Schwarz Algorithm (in both iterative and Krylov accelerated form) for the different interface conditions introduced in Section 4, with respect to mesh and subdomain sizes as well as the fiber orientation (Sect. 6.1 through 6.4). Then, to test the accuracy of the method, we consider the propagation of an action potential during a whole heart cycle (Sects. 6.5 and 6.6) and we compare the solution of a global Bidomain solution with the solution obtained performing only a small number of iterations in the OSA. We consider different geometries: a rectangle, a two dimensional circular slab and a simplified ventricular geometry constituted by a truncated ellipsoid (see [10]).

The system is advanced in time by the semi-implicit scheme (2.6) described in Section 2.1.1, that linearizes the ionic current around the previous time step. In order to focus only on the performance of the Optimized Schwarz Algorithm, in all the simulations presented in this Section we use a fixed time step $\Delta t=0.05 \mathrm{~ms}$, which is small enough to capture the abrupt variations in the transmembrane potential during the action potential. The ionic gating variables are integrated exactly in time after linearization, while the concentration variables are advanced in time with a Forward Euler scheme. The series of test is run with LifeV [1], a C++ Finite Element Library using the Trilinos package AZTECOO [2].

\subsection{Influence of the mesh size}

In this first series of tests the computational domain is the rectangle $\Omega=[0,2] \times[0,1]$ decomposed in the two subdomains $\Omega_{1}=[0,1+L] \times[0,1]$ and $\Omega_{2}=[1,2] \times[0,1]$. The fibers are parallel to the reference axes, with the longitudinal direction set orthogonal to the interface. Both domains are discretized by a uniform triangular grid (we denote with $h$ the mesh size), associated with $\mathbb{P}_{1}$ finite elements. Since at each time step the problems to be solved are linear, we analyze here the convergence to the zero solution in the absence of forcing terms, and starting from a random value on the interface to ensure that all frequencies are present in the error.

We consider both a decomposition without overlap $(L=0)$ and with a minimal overlap of one element $(L=h)$. The iterative algorithm stops when the maximum value between $\left\|\boldsymbol{\lambda}_{1,1}^{p+1}-\boldsymbol{\lambda}_{1,1}^{p}\right\|_{l^{2}(\Gamma)}$ and $\left\|\boldsymbol{\lambda}_{1,2}^{p+1}-\boldsymbol{\lambda}_{1,2}^{p}\right\|_{l^{2}(\Gamma)}$ drops below a given tolerance $\varepsilon=1$.e- 6 . We report in Table 1 the iteration counts for the various interface conditions described in Sections 4.2 and 4.3, as well as for the Classical Schwarz Algorithm (CS). We report the results for different mesh sizes: columns 2 through 9 refer to overlapping decomposition, while columns 10 through 17 refer to non-overlapping decomposition. Within each mesh size, the left column refers to the convergence of the $u$ variable, while the right column refers to the convergence of the $u_{e}$ variable. The most effective interface conditions appear to be the two-sided T1, T4 and T5. We observe that even a minimal overlap is enough 
TABLE 1. Iterative Schwarz algorithm: iteration counts to achieve convergence in $u$ and $u_{e}$ for various mesh sizes and different interface conditions. Left: minimal overlapping $(L=h)$. Right: non-overlapping decomposition $(L=0)$.

\begin{tabular}{|c|c|c|c|c|c|c|c|c|c|c|c|c|c|c|c|c|}
\hline \multirow{3}{*}{$h$} & \multicolumn{8}{|c|}{ Overlapping $(L=h)$} & \multicolumn{8}{|c|}{ Non-overlapping $(L=0)$} \\
\hline & \multicolumn{2}{|c|}{$1 / 48$} & \multicolumn{2}{|c|}{$1 / 64$} & \multicolumn{2}{|c|}{$1 / 96$} & \multicolumn{2}{|c|}{$1 / 128$} & \multicolumn{2}{|c|}{$1 / 48$} & \multicolumn{2}{|c|}{$1 / 64$} & \multicolumn{2}{|c|}{$1 / 96$} & \multicolumn{2}{|c|}{$1 / 128$} \\
\hline & $u$ & $u_{e}$ & $u$ & $u_{e}$ & $u$ & $u_{e}$ & $u$ & $u_{e}$ & $u$ & $u_{e}$ & $u$ & $u_{e}$ & $u$ & $u_{e}$ & $u$ & $u_{e}$ \\
\hline $\mathrm{CS}$ & 27 & 71 & 33 & $\overline{92}$ & 44 & 132 & 54 & 170 & - & - & - & - & - & - & - & - \\
\hline O1 & 11 & 41 & 12 & 47 & 13 & 56 & 14 & 61 & 18 & 88 & 18 & 88 & 18 & 88 & 18 & 88 \\
\hline O2 & 5 & 9 & 5 & 10 & 6 & 11 & 7 & 12 & 177 & 63 & 163 & 59 & 152 & 56 & 149 & 55 \\
\hline O3 & 8 & 26 & 9 & 32 & 11 & 42 & 12 & 48 & 12 & 37 & 12 & 46 & 14 & 57 & 14 & 63 \\
\hline $\mathrm{O} 4$ & 10 & 34 & 11 & 39 & 12 & 47 & 13 & 52 & 14 & 57 & 14 & 63 & 15 & 67 & 15 & 68 \\
\hline $\mathrm{T} 1$ & 6 & 13 & 7 & 14 & 7 & 15 & 8 & 16 & 10 & 20 & 10 & 20 & 10 & 20 & 10 & 20 \\
\hline T2 & 9 & 32 & 11 & 38 & 12 & 48 & 13 & 54 & 13 & 51 & 14 & 60 & 15 & 69 & 16 & 73 \\
\hline T3 & 10 & 37 & 11 & 43 & 13 & 51 & 13 & 56 & 15 & 69 & 16 & 73 & 16 & 76 & 16 & 77 \\
\hline $\mathrm{T} 4$ & 7 & 11 & 6 & 12 & 7 & 14 & 7 & 15 & 19 & 14 & 14 & 15 & 10 & 17 & 9 & 17 \\
\hline T5 & 7 & 12 & 7 & 13 & 7 & 14 & 7 & 15 & 12 & 17 & 10 & 17 & 9 & 18 & 9 & 18 \\
\hline T6 & 9 & 29 & 10 & 35 & 12 & 44 & 12 & 50 & 12 & 45 & 13 & 53 & 14 & 62 & 15 & 66 \\
\hline
\end{tabular}

TABLE 2. Krylov acceleration: iteration counts for various mesh sizes and different algorithms for a nonoverlapping decomposition $(L=0)$.

\begin{tabular}{|l|c|c|c|c|c|c|c|c|c|c|}
\hline$h$ & O1 & O2 & O3 & O4 & T1 & T2 & T3 & T4 & T5 & T6 \\
\hline $1 / 48$ & 20 & 22 & 13 & 16 & 11 & 16 & 18 & 13 & 11 & 15 \\
$1 / 64$ & 20 & 22 & 15 & 17 & 11 & 16 & 18 & 11 & 11 & 16 \\
$1 / 96$ & 20 & 21 & 17 & 18 & 10 & 18 & 19 & 11 & 11 & 17 \\
$1 / 128$ & 20 & 20 & 17 & 18 & 10 & 18 & 19 & 11 & 11 & 18 \\
\hline
\end{tabular}

to significantly improve the convergence of the algorithm, and this is particularly visible for the interface conditions with less effective performance in the non-overlapping case. In general, the convergence of the $u$ variable is significantly faster than the convergence of the $u_{e}$ variable, with one exception. Infact, the interface condition $\mathbf{O} 2$ behaves, as expected, very poorly in a non-overlapping settings, where the $u$ variable converges much slower than the $u_{e}$ one. Besides, in the overlapping case the interface condition $\mathbf{O} 2$ is the most effective. The Classical Schwarz Algorithm is convergent only in the presence of overlap, but appears to be very sensitive to the mesh size, while the Optimized Schwarz Algorithm appears to be more robust with respect to the mesh size, especially with the two-sided conditions T1, T4 and T5.

\subsection{Krylov acceleration}

In this section we consider the use of the Optimized Schwarz Algorithm as a preconditioner to accelerate the convergence of a Krylov method. The computational domain is again the rectangle $\Omega=[0,2] \times[0,1]$ decomposed in the two nonoverlapping subdomains $\Omega_{1}=[0,1] \times[0,1]$ and $\Omega_{M}=[1,2] \times[0,1]$. As in the previous section, we test the convergence to the null solution starting from a random value on the interface. System (5.5) is solved by GMRES, where the matrix-vector product amounts to solve a subproblem in each subdomain. The algorithm stops when the normalized residual drops below 1.e-5. We report in Table 2 the iteration counts for the various interface conditions described in Sections 4.2 and 4.3. The Krylov method appears to be very robust in terms of both the mesh size and the interface conditions. Still, the two-sided conditions T1, T4 and T5 appear to be the most effective ones.

We then investigate the convergence in $u$ and $u_{e}$ by comparing the errors at convergence between two successive iterates $\left\|\boldsymbol{\lambda}_{1,1}^{p+1}-\boldsymbol{\lambda}_{1,1}^{p}\right\|_{l^{2}(\Gamma)}$ and $\left\|\boldsymbol{\lambda}_{1,2}^{p+1}-\boldsymbol{\lambda}_{1,2}^{p}\right\|_{l^{2}(\Gamma)}$, where $\boldsymbol{\lambda}_{1}$ has been introduced in Section 5 . The mesh size is $h=1 / 96$. We can see from Table 3 that the Krylov method, besides a significant speed-up, ensures a 
TABLE 3. Krylov acceleration in a nonoverlapping decomposition $(L=0)$ : iteration counts and relative error at convergence.

\begin{tabular}{|l|c|c|c|c|c|c|}
\hline \multirow{2}{*}{$h=1 / 96$} & \multicolumn{2}{|c|}{ Iteration counts } & \multicolumn{2}{|c|}{$\left\|\boldsymbol{\lambda}_{1,1}^{p+1}-\boldsymbol{\lambda}_{1,1}^{p}\right\|_{l^{2}(\Gamma)}$} & \multicolumn{2}{|c|}{$\left\|\boldsymbol{\lambda}_{1,2}^{p+1}-\boldsymbol{\lambda}_{1,2}^{p}\right\|_{l^{2}(\Gamma)}$} \\
\cline { 2 - 7 }$h$ & Krylov & Iterative & Krylov & Iterative & Krylov & Iterative \\
\hline O1 & 20 & 88 & $1.1847 \mathrm{e}-07$ & $3.1015 \mathrm{e}-10$ & $2.3234 \mathrm{e}-07$ & $9.4846 \mathrm{e}-07$ \\
O2 & 21 & 152 & $8.6393 \mathrm{e}-06$ & $9.9918 \mathrm{e}-07$ & $2.7666 \mathrm{e}-06$ & $2.7231 \mathrm{e}-09$ \\
O3 & 17 & 57 & $1.9402 \mathrm{e}-08$ & $3.1094 \mathrm{e}-10$ & $7.3750 \mathrm{e}-08$ & $9.5069 \mathrm{e}-07$ \\
O4 & 18 & 67 & $5.5186 \mathrm{e}-08$ & $3.0551 \mathrm{e}-10$ & $2.0568 \mathrm{e}-07$ & $9.3419 \mathrm{e}-07$ \\
\hline T1 & 10 & 20 & $9.3953 \mathrm{e}-07$ & $3.1597 \mathrm{e}-09$ & $1.9769 \mathrm{e}-07$ & $6.8538 \mathrm{e}-07$ \\
T2 & 18 & 69 & $8.0154 \mathrm{e}-08$ & $3.1085 \mathrm{e}-10$ & $2.0789 \mathrm{e}-07$ & $9.5061 \mathrm{e}-07$ \\
T3 & 19 & 76 & $1.3719 \mathrm{e}-08$ & $3.0742 \mathrm{e}-10$ & $1.3286 \mathrm{e}-07$ & $9.4012 \mathrm{e}-07$ \\
T4 & 11 & 17 & $9.8231 \mathrm{e}-08$ & $3.1062 \mathrm{e}-09$ & $1.3248 \mathrm{e}-07$ & $6.0135 \mathrm{e}-07$ \\
T5 & 11 & 18 & $8.5966 \mathrm{e}-08$ & $2.6865 \mathrm{e}-09$ & $1.6897 \mathrm{e}-07$ & $6.5841 \mathrm{e}-07$ \\
T6 & 17 & 62 & $2.5537 \mathrm{e}-08$ & $2.8719 \mathrm{e}-10$ & $1.3134 \mathrm{e}-07$ & $8.7807 \mathrm{e}-07$ \\
\hline
\end{tabular}

TABLE 4. Iteration counts for different interface positions in a nonoverlapping decomposition $(L=0)$.

\begin{tabular}{|c|c|c|c|c|c|c|c|c|c|}
\hline$\Omega_{1}=[0, a] \times[0,1]$ & \multicolumn{3}{|c|}{$a=0.5$} & \multicolumn{3}{|c|}{$a=0.75$} & \multicolumn{3}{|c|}{$a=1$} \\
\hline \multirow[b]{2}{*}{$h=1 / 96$} & \multicolumn{2}{|c|}{ Iterative } & \multirow[t]{2}{*}{ Krylov } & \multicolumn{2}{|c|}{ Iterative } & \multirow[t]{2}{*}{ Krylov } & \multicolumn{2}{|c|}{ Iterative } & \multirow[t]{2}{*}{ Krylov } \\
\hline & $u$ & $u_{e}$ & & $u$ & $u_{e}$ & & $u$ & $u_{e}$ & \\
\hline O1 & 18 & 90 & 20 & 18 & 88 & 20 & 18 & 88 & 20 \\
\hline $\mathrm{O} 2$ & 152 & 56 & 21 & 152 & 56 & 21 & 152 & 56 & 21 \\
\hline $\mathrm{O} 3$ & 14 & 59 & 17 & 14 & 57 & 17 & 14 & 57 & 17 \\
\hline $\mathrm{O} 4$ & 15 & 69 & 18 & 15 & 67 & 18 & 15 & 67 & 18 \\
\hline T1 & 10 & 20 & 10 & 10 & 20 & 10 & 10 & 20 & 10 \\
\hline T2 & 15 & 71 & 18 & 15 & 69 & 18 & 15 & 69 & 18 \\
\hline T3 & 16 & 78 & 19 & 16 & 76 & 19 & 16 & 73 & 19 \\
\hline T4 & 10 & 17 & 11 & 10 & 17 & 11 & 10 & 17 & 11 \\
\hline T5 & 9 & 18 & 11 & 9 & 18 & 11 & 9 & 18 & 11 \\
\hline T6 & 14 & 63 & 17 & 14 & 62 & 17 & 14 & 62 & 18 \\
\hline
\end{tabular}

more balanced convergence to $u$ and $u_{e}$, with respect to the iterative version of the Optimized Schwarz Method, where the convergence of the $u$ variable is in general much faster than the convergence of $u_{e}$.

\subsection{Influence of the position of the interface}

In this series of tests we investigate the dependence on the interface position. The computational domain $\Omega=[0,2] \times[0,1]$ is decomposed in the two non overlapping subdomains $\Omega_{1}=[0, a] \times[0,1]$ and $\Omega_{2}=[a, 2] \times[0,1]$, where we choose $a=0.5,0.75,1$. The mesh size is $h=1 / 96$. Since the Classical Schwarz algorithm is not convergent in the absence of overlap, we consider here only the Optimized Schwarz Algorithms with the interface conditions described in Sections 4.2 and 4.3.

We can infer from Table 4 that the Optimized Schwarz Algorithm is very stable with respect to the position of the interface, both in its iterative form and when used as a preconditioner for the a Krylov method to solve system (5.5). 

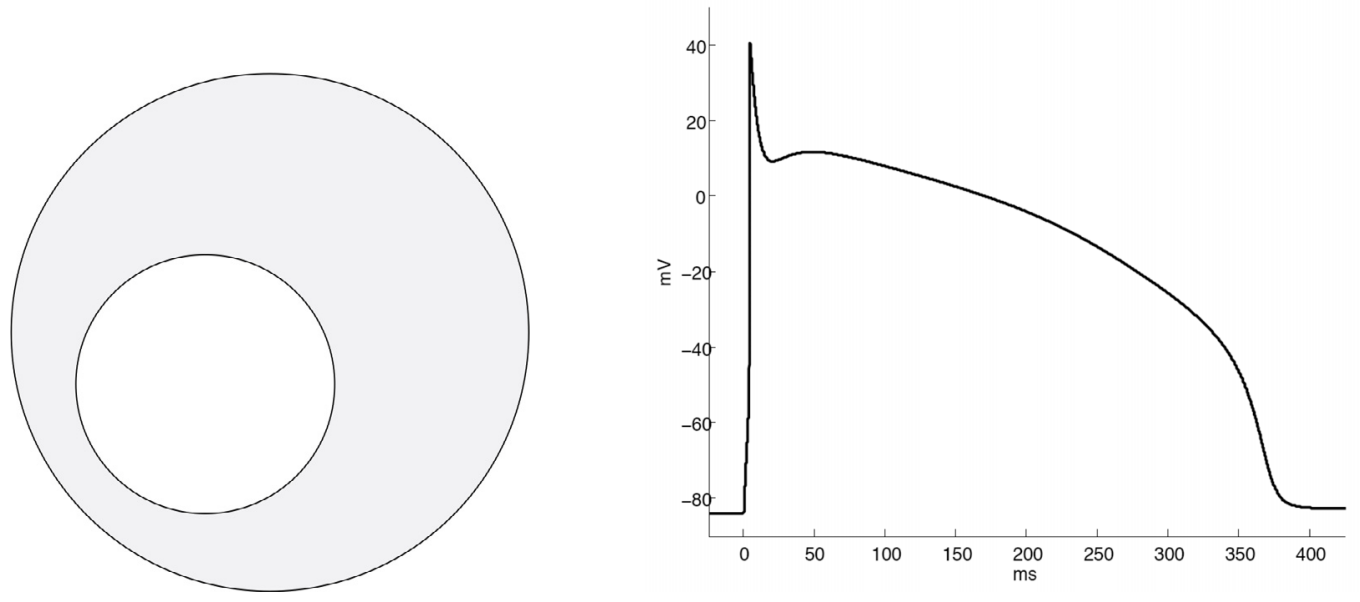

FiguRE 5. Left: the computational domain $\Omega=\left\{(x, y) \in \mathbb{R}^{2} \mid(x-1)^{2}+(y-1)^{2} \leq 1\right\}$, and the two subdomains $\Omega_{1}$ (white) and $\Omega_{2}$ (light grey). Right: time evolution of the action potential in the Luo-Rudy Phase I model.

TABLE 5. Iteration counts: $\Gamma=\left\{(x-0.75)^{2}+(y-0.8)^{2}=0.25\right\}$, while the fibers direction forms an angle $\theta=\pi / 4$ with the cartesian $x$-axis.

\begin{tabular}{|l|l|c|c|c|c|c|c|c|c|c|c|}
\hline \multicolumn{2}{|l|}{} & O1 & O2 & O3 & O4 & T1 & T2 & T3 & T4 & T5 & T6 \\
\hline Iterative & $u$ & 60 & 192 & 48 & 50 & 13 & 52 & 54 & 16 & 16 & 50 \\
& $u_{e}$ & 288 & 167 & 220 & 231 & 20 & 249 & 256 & 18 & 18 & 225 \\
\hline Krylov & 52 & 46 & 47 & 47 & 17 & 48 & 49 & 21 & 21 & 47 \\
\hline
\end{tabular}

\subsection{Influence of the fibers direction}

In this series of tests we investigate the dependence upon the fibers direction. In order to consider all the possible incidence angles between the fibers and the interface, the computational domain is the unit circle centered in (1,1), $\Omega=\left\{(x, y) \in \mathbb{R}^{2} \mid(x-1)^{2}+(y-1)^{2} \leq 1\right\}$, and is decomposed in the two non overlapping subdomains $\Omega_{1}=\left\{(x, y) \in \mathbb{R}^{2} \mid(x-0.75)^{2}+(y-0.8)^{2} \leq 0.25\right\}$ and $\Omega_{2}=\Omega \backslash \Omega_{1}$ (see Fig. 5). The fibers are oriented to form an angle $\theta=\pi / 4$ with the cartesian $x$-axis and the computational domain is discretized by an unstructured triangular grid, that is conform on the interface $\Gamma$, and features a mesh size $h=1.8 \mathrm{e}-2$. Again, we test the convergence to the null solution, and since the Classical Schwarz algorithm is not convergent in the absence of overlap, we consider here only the Optimized Schwarz Algorithms with the different interface conditions described in Sections 4.2 and 4.3.

From Table 5 we can observe that the iteration counts increase significantly for both the iterative version and the Krylov acceleration for all the interface conditions except for the two-sided conditions T1, T4 and $\mathbf{T 5}$, whose effectiveness is confirmed also in this more complex case. In particular, the $\mathbf{T} \mathbf{1}$ interface condition appears to be the most effective in this more general framework when a Krylov method is used.

\subsection{Accuracy}

We now test the accuracy of the Optimized Schwarz Algorithm during the propagation of an action potential in the computational domain used in the previous test of Section 6.4 (Fig. 5, left). During the first millisecond of the simulation we apply a current stimulus $I_{i}^{\mathrm{app}}=I_{\mathrm{e}}^{\mathrm{app}}=50 \mathrm{~mA} / \mathrm{cm}^{3}$ in the circle $(x-0.6)^{2}+(y-0.7)^{2} \leq(0.12)^{2}$. The ionic model used in the simulation is the Luo-Rudy I [35] (see Fig. 5, right, for the time evolution of the action potential in the Luo-Rudy I model). We use a fixed time step $\Delta t=0.05 \mathrm{~ms}$ to focus only on the coupling 

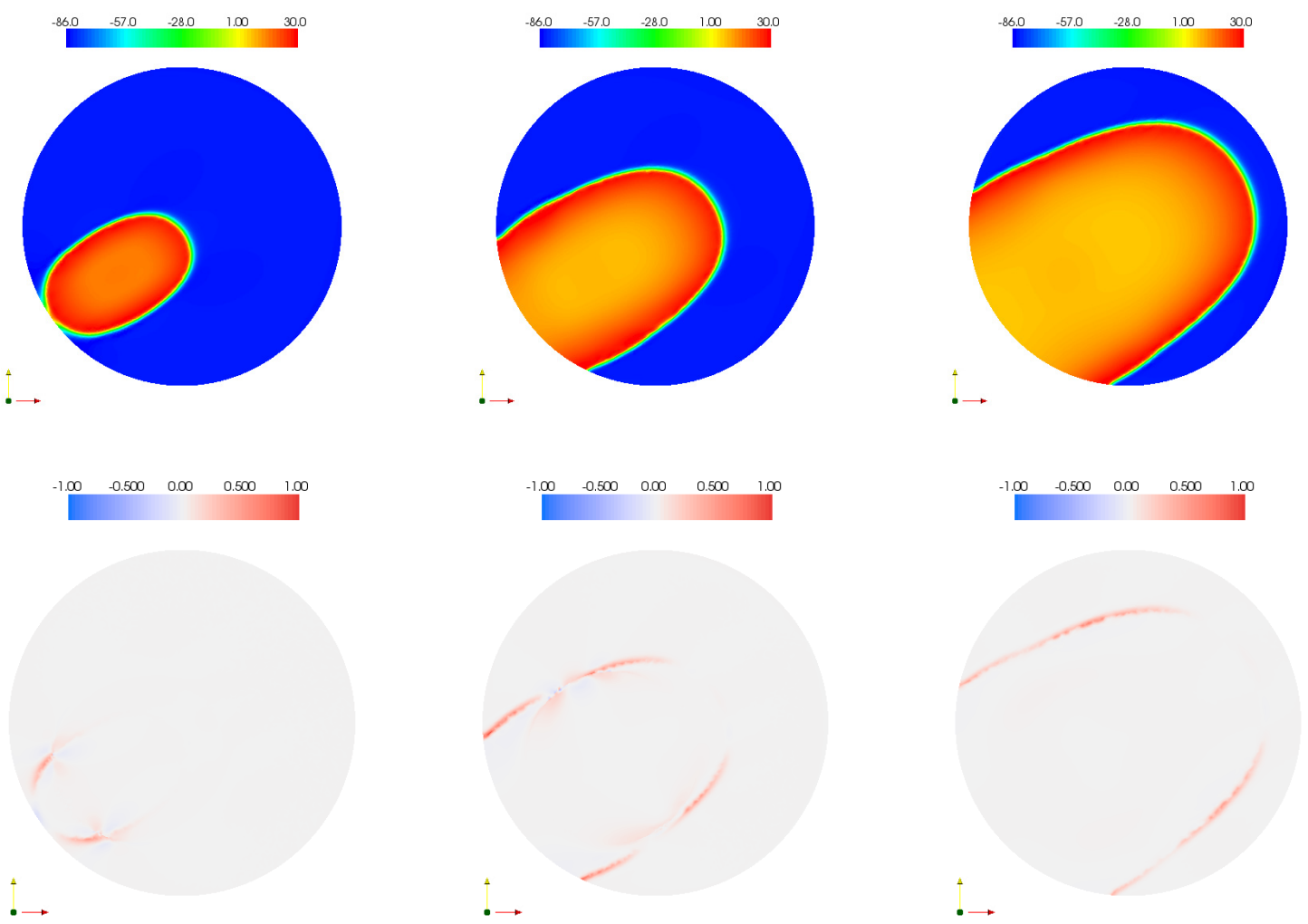

Figure 6 . Action potential $[\mathrm{mV}]$ propagation in a Bidomain simulation. $u_{\text {Bido }}$ and error $u_{\text {Bido }}-$ $u_{\mathrm{OS}}$ along time (left to right, $t=7 \mathrm{~ms}, 13 \mathrm{~ms}, 19 \mathrm{~ms}$ ): $u_{\mathrm{OS}}$ is computed with 2 iterations of the Optimized Schwarz algorithm. Top: action potential; bottom: error.

provided by the Optimized Schwarz Method: time adaptive schemes can be considered as well [44]. Finally, we initialize the OSA at every time step with the transmembrane potential at the previous one.

We report in Figure 6 (top row) the solution at different time levels ( $t=7 \mathrm{~ms}, 13 \mathrm{~ms}, 19 \mathrm{~ms}$ ), during which the front is propagating in $\Omega$ ) obtained from a Bidomain simulation over the whole $\Omega$ (that we denote with $u_{\text {Bido }}$ ), and in the bottom row the error between $u_{\text {Bido }}$ and the solution obtained after 2 iterations of the Optimized Schwarz Method coupled with the two-sided T1 interface condition (that we denote with $u_{\mathrm{OS}}$ ). Figure 7 is analogus to Figure 6 for the extracellular potential $u_{e}$. To assess the relative spatial error during a heartbeat, we compute the quantities

$$
\delta_{u}=\frac{\left\|u_{\mathrm{Bido}}-u_{\mathrm{OS}}\right\|_{L^{2}(\Omega)}}{\left\|u_{\mathrm{Bido}}-u_{\mathrm{rest}}\right\|_{L^{2}(\Omega)}} \quad \delta_{e}=\frac{\left\|u_{e, \text { Bido }}-u_{e, \mathrm{OS}}\right\|_{L^{2}(\Omega)}}{\left\|u_{e, \text { Bido }}\right\|_{L^{2}(\Omega)}},
$$

where $u_{\text {rest }}$ is the resting value of the membrane potential, that are plotted as a function of time in Figure 8 . As expected from the previous analysis on condition T1, the convergence of the Schwartz method for $u_{e}$ is slower than that for $u$, therefore the errors for $u_{e}$ are usually larger the the ones for $u$. However, errors on $u_{e}$ does propagate in time, since the solution $\boldsymbol{u}(\boldsymbol{x}, T)$ at any time $T$ does not depend on $u_{e}(\boldsymbol{x}, t)$, for $t<T$. From Figures 6 and 7 , it can be seen that the errors in $u$ and $u_{e}$ are concentrated, as expected, in the neighborhood of the propagation front, and is confined within a $2 \%$ of the Bidomain solution. There is basically no dissipation due to the Optimized Schwarz Method, and the propagation front is very well captured with a barely appreciable 

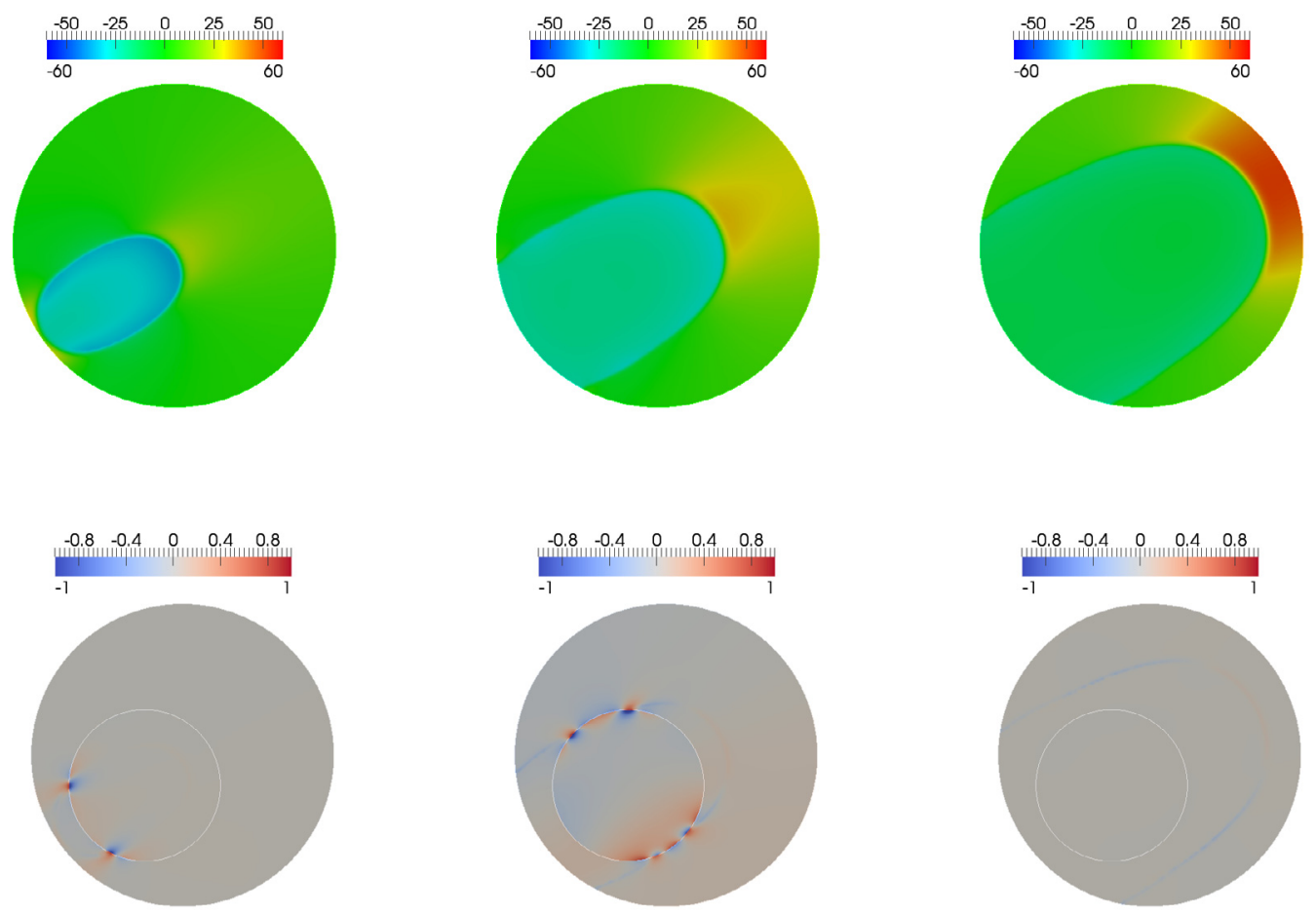

Figure 7. Extracellular potential $[\mathrm{mV}]$ in a Bidomain simulation. $u_{e, \text { Bido }}$ and error $u_{e, \text { Bido }}-$ $u_{e, \mathrm{OS}}$ along time (left to right, $t=7 \mathrm{~ms}, 13 \mathrm{~ms}, 19 \mathrm{~ms}$ ): $u_{\mathrm{OS}}$ is computed with 2 iterations of the Optimized Schwarz algorithm. Top: action potential; bottom: error.

delay even if only 2 iterations of the algorithm are performed. This is very promising in view of a model adaptive strategy: the computational domain can be split into several subdomains, each of which is associated with the Bidomain or the Monodomain model accordingly to an a posteriori estimator such as the one proposed in [36]. The latter is a single parabolic reaction-diffusion equation for the membrane potential $u$, based on a quite unphysical proportionality assumption between the intracellular and extracellular conductivity tensors $\left(\mathbf{D}_{i}=\lambda \mathbf{D}_{e}\right.$, see e.g. [10]). In many applications the Monodomain model is accurate enough to describe the main dynamics of action potential, however, in a few cases (e.g. in presence of pacemaker leads), it may not be able to capture the correct patterns. In this respect, Potse et al. [45] stated that "in absence of applied currents, propagating of action potentials on the scale of a human heart can be studied with a monodomain model". The coupling of the Monodomain model with the Bidomain model in non-symmetric form (2.5) is based on the coupling of the transmembrane potential $u$ [28]. Thus, in such a framework, Figure 6 suggests that running only 2 iterations of the Optimized Schwarz Method, possibly coupled with a time adaptive strategy in the presence of the depolarization front can be a reasonable trade-off between accuracy and efficiency.

\subsection{Action potential propagation in a simplified ventricular geometry}

We conclude our analysis by simulating the action potential propagation in a simplified ventricular geometry, constituted by a truncated ellipsoid [10], with the analytical fibers mapping described therein. As usual we denote with $\Omega$ the computational domain, that we split in the two subdomains $\Omega_{1}$ and $\Omega_{2}$ (Fig. 9). We discretize the computational domain with a tetrahedral mesh, whose average element diameter is $0.6 \mathrm{~mm}$. We use a fixed 

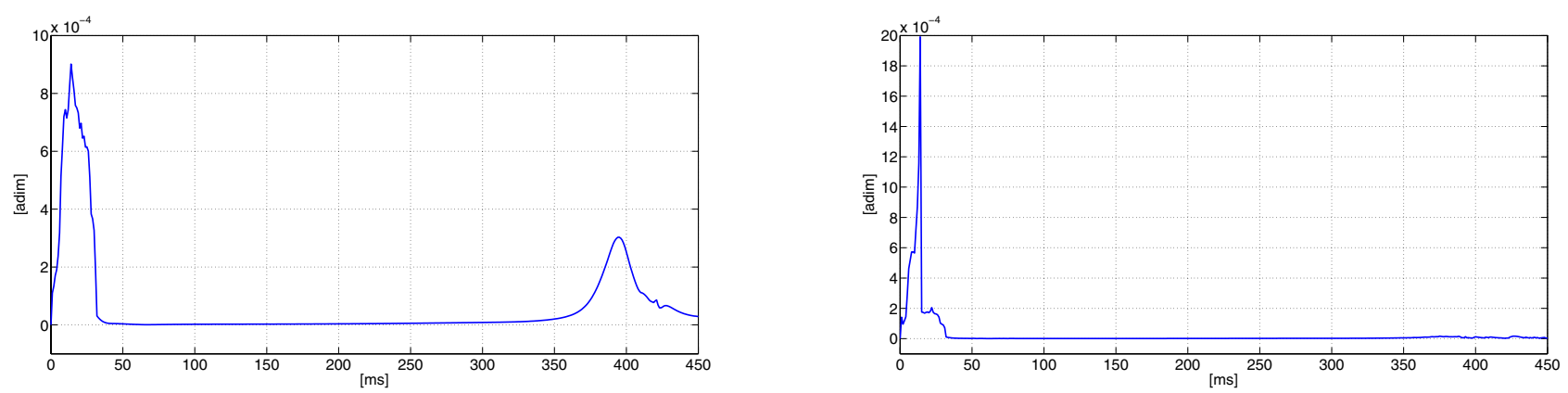

FiguRE 8. Relative spatial error, during an heartbeat, for the membrane and extracellular potentials as a function of time: $\delta_{u}$ (left) and $\delta_{e}$ (right).
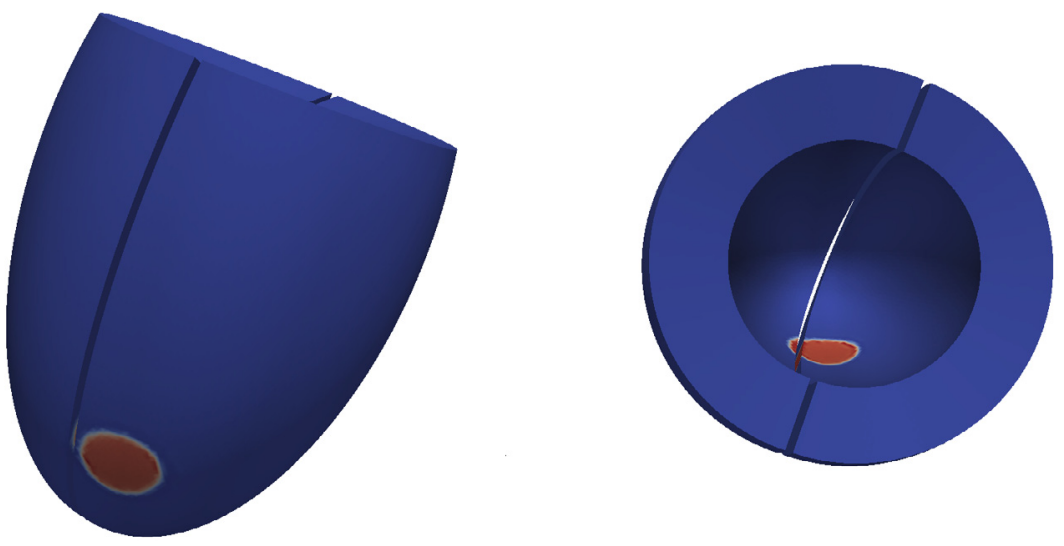

Figure 9. Frontal and transversal views of the computational domain decomposed in two domains. The red spot is indicates the stimulus region.

time step $\Delta t=0.05 \mathrm{~ms}$ and we run a simulation for $450 \mathrm{~ms}$. The ionic model used in the simulation is the Luo-Rudy I [35]. We apply a current stimulus $I_{i}^{\mathrm{app}}=I_{\mathrm{e}}^{\mathrm{app}}=50 \mathrm{~mA} / \mathrm{cm}^{3}$ during the first millisecond in the ball $(x-1.5)^{2}+(y-0.5)^{2}+(z-3)^{2} \leq(0.7)^{2}$.

We report in Figure 10 (first column) the membrane potential at different times $(t=7 \mathrm{~ms}$ in the first row and $t=25 \mathrm{~ms}$ in the second row) computed with the Krylov accelerated version of the Optimized Schwarz algorithm and interface condition T1. We also plot the errors between the global Bidomain solution and the solution obtained with the Optimized Schwarz Algorithm. In the second and third column the OSA is run for only 2 and 4 iterations respectively. In the fourth column, the Krylov algorithm is run until convergence with a coarse tolerance $(\varepsilon=1 e-3)$. On average, the Krylov algorithm takes 3.5 iterations for reaching the given tolerance $\varepsilon$ when the action potential front is traveling through the interface, whereas the iterative algorithm takes about 12 iterations to reach the same tolerance. As for the transmembrane potential $u$, the error is well concentrated around the propagation front. Four iterations of the OSA algorithm are sufficient to confine the

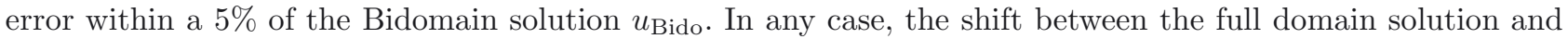
the Domain Decomposition solution is moderate. Figure 11 shows the extracellular potential for $t=7 \mathrm{~ms}$ and $t=25 \mathrm{~ms}$. In this case the error on $u_{e}$ is much larger than the error in $u$ when only 2 or 4 iterations of the OSA method are performed. This is consistent with the fact that the convergence of $u_{e}$ is much slower than that of $u$ for condition T1. Also we notice that the error on $u_{e}$ is not localized near the front. As already observed in the 2D simulations, the Krylov algorithm features a more balanced convergence for $u$ and $u_{e}$. This is evident 

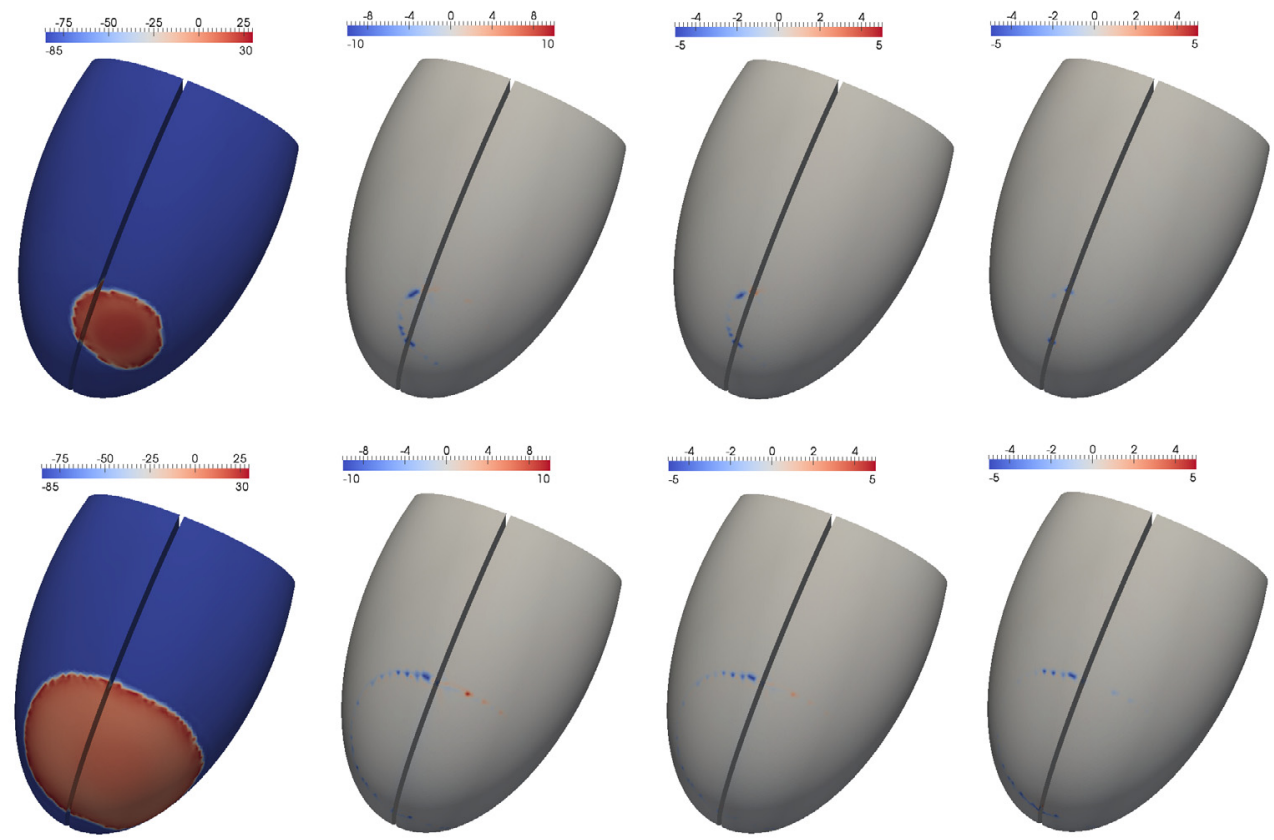

Figure 10. Action potential propagation in a Bidomain simulation $u_{\text {Bido }}$ (first column) and errors $u_{\text {Bido }}-u_{\text {OS }}$ for different algorithms and stopping criteria to compute $u_{\text {OS }}$ (second column: Krylov, T1, $\varepsilon=1 e-3$; third column: T1, 2 iterations; fourth column: T1, 4 iterations). Top: $t=7 \mathrm{~ms}$, bottom: $t=25 \mathrm{~ms}$.

also from Figure 12 which shows the relative error in space as a function of time, as introduced in (6.1). It is worth noting that the error on $u$ is smaller when the problem is solved with 4 iterations than with the Krylov method. On the other hand the error on $u_{e}$ is much better when using Krylov method rather than the iterative method.

\section{Conclusions}

We analyzed Schwarz methods applied to the Bidomain system in electrocardiology. The Classical Schwarz requires overlap to converge, while the Optimized Schwarz Algorithm introduced here ensure convergence also in the absence of overlap. Moreover, in the presence of overlap the Optimized Schwarz Algorithm performs better than the classical Schwarz one. We analyzed and tested one-sided and two-sided Robin interface conditions. Twosided conditions appear to be in general more effective, and we empirically devised a parameters combination that is robust with respect to both the mesh size and the fibers directions.

Despite the higher accuracy allowed by the reduced size of the problem, each iteration of the Optimized Schwarz Algorithm (both in iterative form and in preconditioner form for a Krylov method) requires the solution of two ( $N$ in the framework of a more general decomposition in $N$ subdomains) costly Bidomain subproblems, even if effective preconditioning strategies are used within the subdomains. A further improvement relies in a model adaptive strategy, where the computational domain is split into several subdomains and the Bidomain model is solved only in regions where its accuracy is really required, while the cheaper Monodomain model is employed in the rest of the domain. The latter is a simpler, albeit less accurate, model relying on an unrealistic proportionality assumption between the intracellular and extracellular conductivities and featuring a single parabolic equation for the transmembrane potential, see e.g. [10]. The subdomains are coupled through 

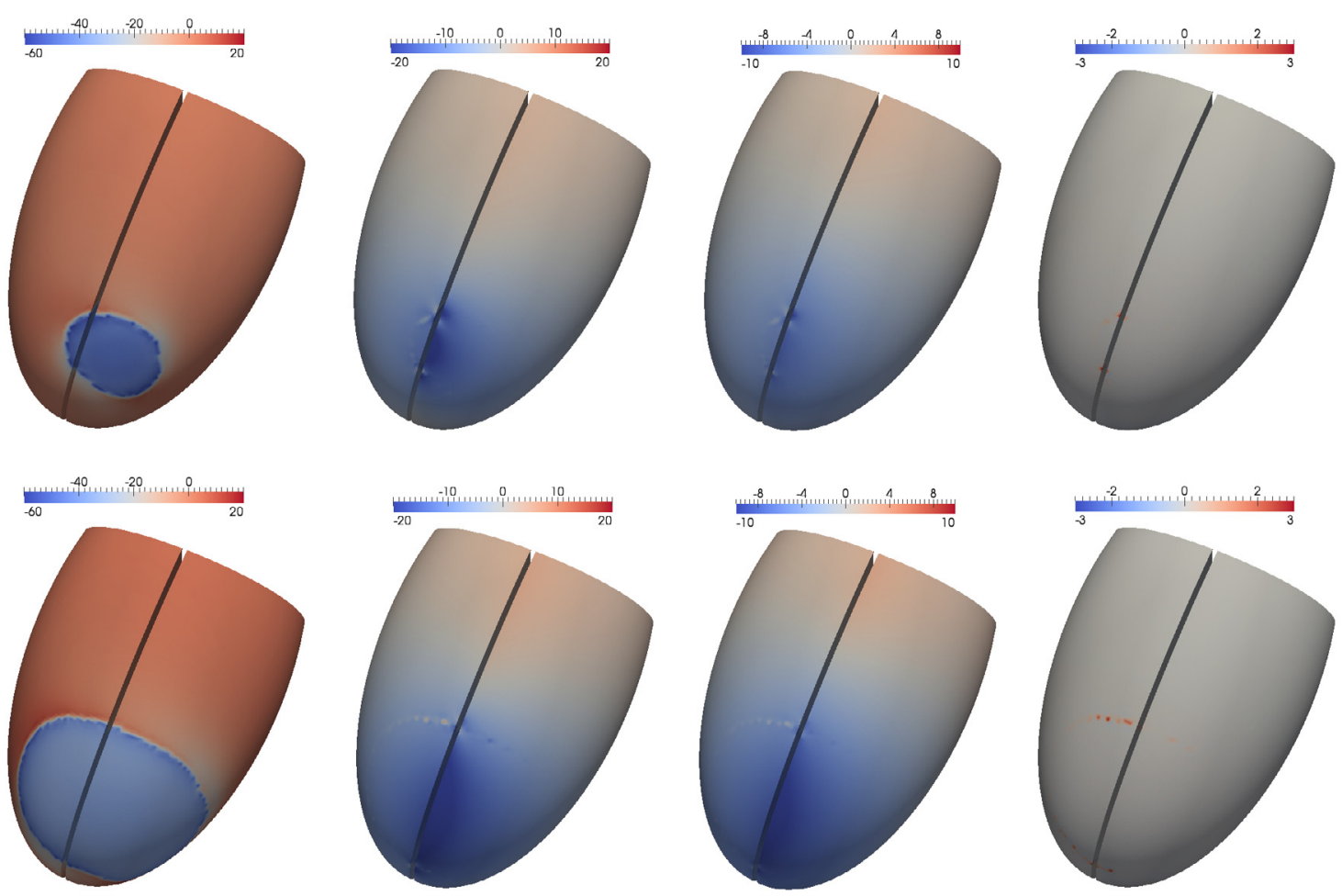

Figure 11. Extracellular potential in a Bidomain simulation $u_{e, \text { Bido }}$ (first column) and errors $u_{e, \text { Bido }}-u_{e, \text { OS }}$ for different algorithms and stopping criteria to compute $u_{e, \text { OS }}$ (second column: Krylov, T1, $\varepsilon=1 e-3$; third column: T1, 2 iterations; fourth column: T1, 4 iterations). Top: $t=7 \mathrm{~ms}$, bottom: $t=25 \mathrm{~ms}$.
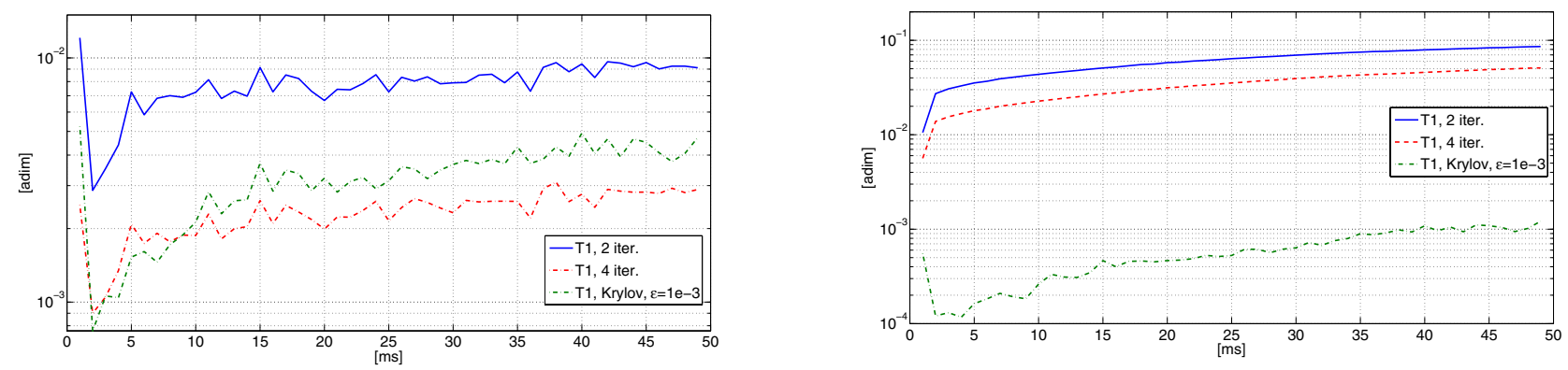

Figure 12. Relative spatial error as a function of time for different OSA algorithms during a heartbeat: $\delta_{u}$ (left) and $\delta_{e}$ (right).

the Optimized Schwarz Method along the interfaces (the one introduced in [28] for a Bidomain/Monodomain coupling, the one proposed in this paper for a Bidomain/Bidomain coupling). The model adaptive approach is currently under investigation and will be the subject of a forthcoming paper. 


\section{REFERENCES}

[1] LifeV software. http://www.LifeV.org.

[2] Trilinos software. http://trilinos.sandia.gov.

[3] A. Alonso-Rodriguez and L. Gerardo-Giorda, New non-overlapping domain decomposition methods for the time-harmonic Maxwell system. SIAM J. Sci. Comput. 28 (2006) 102-122.

[4] P. Bochev and R. Lehouc, On the finite element solution of the pure Neumann problem. SIAM Rev. 47 (2005) 50-66.

[5] T.F. Chan and T.P. Mathew, Domain decomposition algorithms, in Acta Numerica 1994. Cambridge University Press (1994) 61-143.

[6] P. Charton, F. Nataf and F. Rogier, Méthode de décomposition de domaine pour l'équation d'advection-diffusion. C. R. Acad. Sci. 313 (1991) 623-626.

[7] P. Chevalier and F. Nataf, Symmetrized method with optimized second-order conditions for the Helmholtz equation, in Domain decomposition methods (Boulder, CO, 1997). Amer. Math. Soc. 10 (1998) 400-407.

[8] R.H. Clayton, O.M. Bernus, E.M. Cherry, H. Dierckx, F.H. Fenton, L. Mirabella, A.V. Panfilov, F.B. Sachse, G. Seemann and H. Zhang, Models of cardiac tissue electrophysiology: Progress, challenges and open questions. Progr. Bioph. Molec. Biol. 104 (2011) 22-48.

[9] R.H. Clayton and A.V. Panfilov, A guide to modelling cardiac electrical activity in anatomically detailed ventricles. Progr. Bioph. Molec. Biol. 96 (2008) 19-43.

[10] P. Colli Franzone and L.F. Pavarino, A parallel solver for reaction-diffusion systems in computational electrocardiology. Math. Models Methods Appl. Sci. 14 (2004) 883-911.

[11] P. Colli Franzone, L. Pavarino and G. Savaré, Computational electrocardiology: mathematical and numerical modeling, in Complex Systems in Biomedicine - A. Quarteroni, edited by L. Formaggia and A. Veneziani. Springer, Milan (2006).

[12] P. Colli Franzone and G. Savaré, Degenerate evolution systems modeling the cardiac electric field at micro and macroscopic level, in Evolution Equations, Semigroups and Functional Analysis, edited by A. Lorenzi and B. Ruf. Birkhauser (2002) 49-78.

[13] Q. Deng, An analysis for a nonoverlapping domain decomposition iterative procedure. SIAM J. Sci. Comput. 18 (1997) $1517-1525$.

[14] V. Dolean and F. Nataf, An Optimized Schwarz Algorithm for the compressible Euler equations, in Domain Decomposition Methods in Science and Engineering XVI (Proceedings of the DD16 Conference). Springer-Verlag (2007) 173-180.

[15] V. Dolean, M.J. Gander and L. Gerardo-Giorda, Optimized Schwarz Methods for Maxwell's equations. SIAM J. Sci. Comput. 31 (2009) 2193-2213.

[16] O. Dubois, Optimized Schwarz Methods with Robin conditions for the Advection-Diffusion Equation, in Domain Decomposition Methods in Science and Engineering XVI (Proceedings of the DD16 Conference). Springer-Verlag (2007) $181-188$.

[17] B. Engquist and H.-K. Zhao, Absorbing boundary conditions for domain decomposition. Appl. Numer. Math. 27 (1998) 341-365.

[18] E. Faccioli, F. Maggio, A. Quarteroni and A. Tagliani, Spectral domain decomposition methods for the solution of acoustic and elastic wave propagation. Geophys. 61 (1996) 1160-1174.

[19] E. Faccioli, F. Maggio, A. Quarteroni and A. Tagliani, 2d and 3d elastic wave propagation by pseudo-spectral domain decomposition method. J. Seismology 1 (1997) 237-251.

[20] M.J. Gander, Optimized Schwarz methods. SIAM J. Numer. Anal. 44 (2006) 699-731.

[21] M.J. Gander and L. Halpern, Méthodes de relaxation d'ondes pour l'équation de la chaleur en dimension 1. C. R. Acad. Sci. Paris, Sér. I 336 (2003) 519-524.

[22] M.J. Gander, L. Halpern and F. Magoulès, An optimized Schwarz method with two-sided Robin transmission conditions for the Helmholtz equation. Int. J. Numer. Meth. Fluids 55 (2007) 163-175.

[23] M.J. Gander, L. Halpern and F. Nataf, Optimal Schwarz waveform relaxation for the one dimensional wave equation. SIAM J. Numer. Anal. 41 (2003) 1643-1681.

[24] M.J. Gander, F. Magoulès and F. Nataf, Optimized Schwarz methods without overlap for the Helmholtz equation. SIAM J. Sci. Comput. 24 (2002) 38-60.

[25] L. Gerardo-Giorda, L. Mirabella, M. Perego and A. Veneziani, A model adaptive strategy for computational electrocardiology. Domain Decomposition Methods in Science and Engineering XXI (Proceedings of the DD21 Conference). Springer-Verlag. To appear (2013).

[26] L. Gerardo-Giorda, L. Mirabella, F. Nobile, M. Perego and A. Veneziani, A model-based block-triangular preconditioner for the Bidomain system in electrocardiology. J. Comput. Phys. 228 (2009) 3625-3639.

[27] L. Gerardo-Giorda, F. Nobile and C. Vergara, Analysis and optimization of Robin-Robin partitioned procedures in FluidStructure Interaction problems. SIAM J. Numer. Anal. 48 (2010) 2091-2116.

[28] L. Gerardo-Giorda, M. Perego and A. Veneziani, Optimized Schwarz coupling of Bidomain and Monodomain models in electrocardiology. ESAIM: M2AN 45 (2011) 309-334.

[29] T. Hagstrom, R.P. Tewarson and A. Jazcilevich, Numerical experiments on a domain decomposition algorithm for nonlinear elliptic boundary value problems. Appl. Math. Lett. 1 (1988) 299-302.

[30] C.S. Henriquez, Simulating the electrical behavior of cardiac tissue using the Bidomain model. Crit. Rev. Biomed. Eng. 21 (1993) $1-77$. 
[31] C. Japhet, F. Nataf and F. Rogier, The optimized order 2 method: Application to convection-diffusion problems. Future Gener. Comp. Syst. 18 (2001) 17-30.

[32] S. Linge, J. Sundnes, M. Hanslien, G.T. Lines and A. Tveito, Numerical solution of the bidomain equations. Phil. Trans. R. Soc. A. 367 (2009) 1931-1950.

[33] G.T. Lines, M.L. Buist, P. Grottum, A.J. Pullan, J. Sundnes and A. Tveito, Mathematical models and numerical methods for the forward problem in cardiac electrophysiology. Comput. Vis. Sci. 5 (2003) 215-239.

[34] P.-L. Lions, On the Schwarz alternating method. III: a variant for nonoverlapping subdomains, in Third International Symposium on Domain Decomposition Methods for Partial Differential Equations, held in Houston, Texas, edited by T.F. Chan, R. Glowinski, J. Périaux and O. Widlund, SIAM Philadelphia, PA (1990).

[35] L. Luo and Y. Rudy, A model of the ventricular cardiac action potential: depolarization, repolarization and their interaction. Circ. Res. 68 (1991) 1501-1526.

[36] L. Mirabella, F. Nobile and A. Veneziani, An a posteriori error estimator for model adaptivity in electrocardiology. Comput. Methods Appl. Mech. Eng. 200 (2011) 2727-2737.

[37] M. Munteanu, L.F. Pavarino and S. Scacchi, A scalable Newton-Krylov-Schwarz method for the Bidomain reaction-diffusion system. SIAM J. Sci. Comput. 3 (2009) 3861-3883.

[38] F. Nataf and F. Rogier, Factorization of the convection-diffusion operator and the Schwarz algorithm. $M^{3} A S \mathbf{5}$ (1995) 67-93.

[39] L.F. Pavarino and S. Scacchi, Multilevel additive Schwarz preconditioners for the Bidomain reaction-diffusion system. SIAM J. Sci. Comput. 31 (2008) 420-443.

[40] L.F. Pavarino and S. Scacchi, Parallel Multilevel Schwarz and block preconditioners for the Bidomain parabolic-parabolic and parabolic-elliptic formulations. SIAM J. Sci. Comput. 33 (2011) 1897-1919.

[41] M. Pennacchio and V. Simoncini, Efficient algebraic solution of reaction-diffusion systems for the cardiac excitation process. J. Comput. Appl. Math. 145 (2002) 49-70.

[42] M. Pennacchio and V. Simoncini, Algebraic multigrid preconditioners for the Bidomain reaction-diffusion system. Appl. Numer. Math. 59 (2009) 3033-3050.

[43] M. Pennacchio and V. Simoncini, Non-symmetric Algebraic Multigrid Preconditioners for the Bidomain reaction-diffusion system, in Numerical Mathematics and Advanced Applications, ENUMATH 2009, Part 2 (2010) 729-736.

[44] M. Perego and A. Veneziani, An efficient generalization of the Rush-Larsen method for solving electro-physiology membrane equations. ETNA 35 (2009) 234-256.

[45] M. Potse, B. Dubé, J. Richer and A. Vinet, A comparison of Monodomain and Bidomain Reaction-Diffusion models for Action Potential Propagation in the Human Heart. IEEE Trans. Biomed. Eng. 53 (2006) 2425-2435,

[46] A.J. Pullan, M.L. Buist and L.K. Cheng, Mathematical Modelling the Electrical Activity of the Heart. World Scientific, Singapore (2005).

[47] A. Quarteroni and A. Valli, Domain Decomposition Methods for Partial Differential Equations. Oxford Science Publications (1999).

[48] B.J. Roth, Action potential propagation in a thick strand of cardiac muscle. Circ. Res. 68 (1991) $162-173$.

[49] F.B. Sachse, Computational Cardiology. Springer, Berlin (2004).

[50] S. Scacchi, A hybrid multilevel Schwarz method for the Bidomain model. Comput. Methods Appl. Mech. Eng. 197 (2008) 4051-4061.

[51] B.F. Smith, P.E. Bjørstad and W. Gropp. Domain Decomposition: Parallel Multilevel Methods for Elliptic Partial Differential Equations. Cambridge University Press (1996).

[52] J. Sundnes, G.T. Lines and A. Tveito, An operator splitting method for solving the bidomain equations coupled to a volume conductor model for the torso. Math. Biosci. 194 (2005) 233-248.

[53] A. Toselli, Overlapping Schwarz methods for Maxwell's equations in three dimensions. Numer. Math. 86 (2000) $733-752$.

[54] A. Toselli and O. Widlund, Domain Decomposition Methods - Algorithms and Theory. Springer Ser. Comput. Math. 34 (2004).

[55] M. Veneroni, Reaction-diffusion systems for the macroscopic Bidomain model of the cardiac electric field. Nonlinear Anal. Real World Appl. 10 (2009) 849-868.

[56] E.J. Vigmond, F. Aguel and N.A. Trayanova, Computational techniques for solving the Bidomain equations in three dimensions. IEEE Trans. Biomed. Eng. 49 (2002) 1260-1269.

[57] E.J. Vigmond, R. Weber dos Santos, A.J. Prassl, M. Deo and G. Plank, Solvers for the caridac Bidomain equations. Prog. Biophys. Mol. Biol. 96 (2008) 3-18.

[58] R. Weber dos Santos, G. Planck, S. Bauer and E.J. Vigmond, Parallel multigrid preconditioner for the cardiac Bidomain model. IEEE Trans. Biomed. Eng. 51 (2004) 1960-1968.

[59] J. Xu and J. Zou, Some nonoverlapping domain decomposition methods. SIAM Review 40 (1998) 857-914.

[60] J. Xu, Iterative methods by space decomposition and subspace correction. SIAM Review 34 (1992) 581-613. 